\title{
Efficient Palladium-Catalyzed Homocoupling Reaction and Sonogashira Cross-Coupling Reaction of Terminal Alkynes under Aerobic Conditions
}

\author{
Jin-Heng Li, * Yun Liang, and Ye-Xiang Xie \\ Key Laboratory of Chemical Biology \& Traditional Chinese Medicine Research, \\ College of Chemistry and Chemical Engineering, Hunan Normal University,
}

Changsha 410081, China

jhli@,hunnu.edu.cn

\section{Supporting Information}

\section{List of Contents}
(A) Typical experimental procedure
(B) Analytical data for 2 and 4-10
S2-7
(C) References
S7-8
(D) Spectra
S9-46 


\section{(A) Typical experimental procedure}

(1) Typical Experimental Procedure for the Palladium-Catalyzed

Homocoupling of Alkynes. A mixture of alkyne 1 (1 mmol), $\mathrm{Pd}(\mathrm{OAc})_{2}(2.0 \mathrm{~mol} \%)$, $\mathrm{CuI}(2.0 \mathrm{~mol} \%)$, DABCO (3 equiv), and $\mathrm{MeCN}(5 \mathrm{~mL})$ was stirred at room temperature for desired time until complete consumption of starting material as judged by TLC and GC analysis. After usual workup, the residue was purified by flash column chromatography to give $\mathbf{2}$ (hexane or hexane/ethyl acetate).

\section{(2) Typical Experimental Procedure for $\mathrm{Pd}(\mathrm{OAc})_{2}$-Catalyzed Sonogashira}

Cross-Coupling Reactions. Firstly, $\mathrm{Pd}(\mathrm{OAc})_{2}(4.5 \mathrm{mg}, 2.0 \mathrm{~mol} \%)$ was dissolved in $\mathrm{MeCN}(200 \mathrm{~mL})$. Then the indicated amount of $\mathrm{Pd}(\mathrm{OAc})_{2}$ acetonitrile solution was added to a mixture of alkyne 1 (the indicated amount in Table 4), ArX 3 (1 mmol), DABCO (3 equiv), and $\mathrm{MeCN}(5 \mathrm{~mL})$. Then the mixture was stirred at the indicated reaction temperature for desired time until complete consumption of starting material as judged by TLC and GC analysis. After the mixture was filtered and evaporated, the residue was purified by flash column chromatography (hexane or hexane/ethyl acetate) to afford the desired coupled products $\mathbf{4 - 1 0}$.

(B) Analytical data for $2^{1-4}$ and 4-10 ${ }^{5-13}$ :

\section{1,4-Diphenyl buta-1,3-diyne (2a)}

${ }^{1} \mathrm{H}$ NMR $\left(400 \mathrm{MHz}, \mathrm{CDCl}_{3}\right) \delta: 7.54(\mathrm{~d}, J=7.4 \mathrm{~Hz}, 4 \mathrm{H}), 7.38-7.34(\mathrm{~m}, 6 \mathrm{H}) ;{ }^{13} \mathrm{C}$ NMR (100 MHz, $\left.\mathrm{CDCl}_{3}\right) \delta: 132.8,129.6,128.8,122.1,81.9$, 74.2. LRMS (EI, $\left.20 \mathrm{eV}\right)$ $m / z(\%): 202\left(\mathrm{M}^{+}, 100\right)$. 


\section{1,4-Bis(p-methylphenyl) buta-1,3-diyne (2b)}

${ }^{1} \mathrm{H} \mathrm{NMR}\left(400 \mathrm{MHz}, \mathrm{CDCl}_{3}\right) \delta: 7.41(\mathrm{~d}, J=8.0 \mathrm{~Hz}, 4 \mathrm{H}), 7.14(\mathrm{~d}, J=8.0 \mathrm{~Hz}, 4 \mathrm{H})$, $2.36(\mathrm{~s}, 6 \mathrm{H}) ;{ }^{13} \mathrm{C} \mathrm{NMR}\left(100 \mathrm{MHz}, \mathrm{CDCl}_{3}\right) \delta: 139.5,132.4,129.2,118.8,81.5,73.4$ 21.6. LRMS (EI, $20 \mathrm{eV}) \mathrm{m} / z(\%): 230\left(\mathrm{M}^{+}, 100\right)$.

\section{1,4-Bis(p-methoxyphenyl) buta-1,3-diyne (2c)}

${ }^{1} \mathrm{H}$ NMR $\left(400 \mathrm{MHz}, \mathrm{CDCl}_{3}\right) \delta: 7.46(\mathrm{~d}, J=8.8 \mathrm{~Hz}, 4 \mathrm{H}), 6.85(\mathrm{~d}, J=8.8 \mathrm{~Hz}, 4 \mathrm{H})$, $3.82(\mathrm{~s}, 6 \mathrm{H}) ;{ }^{13} \mathrm{C} \mathrm{NMR}\left(100 \mathrm{MHz}, \mathrm{CDCl}_{3}\right) \delta: 160.2,134.0,114.1,113.9,81.2,72.9$, 55.3. LRMS (EI, $20 \mathrm{eV}) \mathrm{m} / z(\%): 262\left(\mathrm{M}^{+}, 100\right)$.

\section{1,4-Bis(trifloromethylphenyl) buta-1,3-diyne (2d)}

${ }^{1} \mathrm{H}$ NMR $\left(400 \mathrm{MHz}, \mathrm{CDCl}_{3}\right) \delta: 7.65(\mathrm{~d}, J=8.4 \mathrm{~Hz}, 4 \mathrm{H}), 7.62(\mathrm{~d}, J=8.4 \mathrm{~Hz}, 4 \mathrm{H})$;

${ }^{13} \mathrm{C}$ NMR $\left(100 \mathrm{MHz}, \mathrm{CDCl}_{3}\right) \delta: 132.8,131.2,130.9,125.5,125.4,125.2,125.0,122.3$, 81.0, 75.6. MS m/z (\%): $182\left(\mathrm{M}^{+}, 100\right), 164$ (53), 92 (40), 75 (13). LRMS (EI, $20 \mathrm{eV}$ ) $m / z(\%): 338\left(\mathrm{M}^{+}, 100\right)$.

\section{1,4-Bis(2-pyridine)buta-1,3-diyne (2e)}

${ }^{1} \mathrm{H}$ NMR (400 MHz, $\left.\mathrm{CDCl}_{3}\right) \delta: 8.63(\mathrm{~d}, J=4.4 \mathrm{~Hz}, 2 \mathrm{H}), 7.71(\mathrm{t}, J=7.6 \mathrm{~Hz}, 2 \mathrm{H})$, $7.56(\mathrm{~d}, J=8.4 \mathrm{~Hz}, 2 \mathrm{H}), 7.31(\mathrm{t}, J=4.8 \mathrm{~Hz}, 2 \mathrm{H}) ;{ }^{13} \mathrm{C} \mathrm{NMR}\left(100 \mathrm{MHz}, \mathrm{CDCl}_{3}\right) \delta$ : 150.3, 141.8, 134.3, 128.4, 123.8, 80.8, 73.2; LRMS (EI, $20 \mathrm{eV}) \mathrm{m} / z(\%): 204\left(\mathrm{M}^{+}\right.$ $100)$. 


\section{Tetradeca-6,8-diyne (2f)}

${ }^{1} \mathrm{H}$ NMR $\left(400 \mathrm{MHz}, \mathrm{CDCl}_{3}\right) \delta: 2.25(\mathrm{t}, J=7.2 \mathrm{~Hz}, 4 \mathrm{H}), 1.54-1.46(\mathrm{~m}, 4 \mathrm{H})$, $1.39-1.28(\mathrm{~m}, 8 \mathrm{H}), 0.89(\mathrm{t}, J=7.2 \mathrm{~Hz}, 6 \mathrm{H}) ;{ }^{13} \mathrm{C} \mathrm{NMR}\left(100 \mathrm{MHz}, \mathrm{CDCl}_{3}\right) \delta: 77.5$, 65.2, 31.0, 28.0, 22.2, 19.2, 13.9. LRMS (EI, $20 \mathrm{eV}) \mathrm{m} / z(\%): 190\left(\mathrm{M}^{+}, 1\right), 105$ (57), 91 (100), 79 (49), 67 (31), 41 (27).

\section{Icosa-9,11-diyne (2g)}

${ }^{1} \mathrm{H}$ NMR (400 MHz, $\left.\mathrm{CDCl}_{3}\right) \delta: 2.27(\mathrm{t}, J=7.2 \mathrm{~Hz}, 4 \mathrm{H}), 1.57-1.50(\mathrm{~m}, 4 \mathrm{H})$, $1.43-1.38(\mathrm{~m}, 4 \mathrm{H}), 1.34-1.20(\mathrm{~m}, 16 \mathrm{H}), 0.90(\mathrm{t}, J=6.4 \mathrm{~Hz}, 6 \mathrm{H}) ;{ }^{13} \mathrm{C}$ NMR $(100 \mathrm{MHz}$, $\left.\mathrm{CDCl}_{3}\right) \delta:$ 77.5, 65.2, 31.8, 29.1, 29.0, 28.8, 28.3, 22.6, 19.2, 14.1. LRMS (EI, $\left.20 \mathrm{eV}\right)$ $m / z(\%): 182\left(\mathrm{M}^{+}, 100\right), 164(53), 92(40), 75$ (13).

\section{2,2,7,7-Tetramethyl octa-3,5-diyne (2h)}

${ }^{1} \mathrm{H}$ NMR (400 MHz, $\left.\mathrm{CDCl}_{3}\right) \delta: 1.23(\mathrm{~s}, 18 \mathrm{H}) ;{ }^{13} \mathrm{C} \mathrm{NMR}\left(100 \mathrm{MHz}, \mathrm{CDCl}_{3}\right) \delta:$ 86.3, 63.6, 30.6, 28.0. LRMS (EI, $20 \mathrm{eV}) \mathrm{m} / z(\%): 162\left(\mathrm{M}^{+}, 8\right), 161$ (25), $133(37)$, 119 (55), 91 (100), 79 (59), 67 (57), 41 (50).

\section{1,4-Bis(cyclohex-1-enyl) buta-1,3-diyne (2i)}

${ }^{1} \mathrm{H}$ NMR $\left(400 \mathrm{MHz}, \mathrm{CDCl}_{3}\right) \delta: 6.25(\mathrm{t}, J=4.0 \mathrm{~Hz}, 2 \mathrm{H}), 2.12-2.10(\mathrm{~m}, 8 \mathrm{H})$, 1.66-1.57 (m, 8H); ${ }^{13} \mathrm{C}$ NMR (100 MHz, $\left.\mathrm{CDCl}_{3}\right) \delta: 138.0,119.9,82.7,71.5,28.6$, 25.8, 22.1, 21.3. LRMS (EI, $20 \mathrm{eV}) \mathrm{m} / z(\%): 210\left(\mathrm{M}^{+}, 3\right), 111(20), 85$ (71), $71(95)$, 57 (100), 41 (69). 


\section{1,4-Bis(1-hydroxycyclohexyl) buta-1,3-diyne (2j)}

${ }^{1} \mathrm{H}$ NMR (400 MHz, $\left.\mathrm{CDCl}_{3}\right) \delta: 1.95-1.91(\mathrm{~m}, 4 \mathrm{H}), 1.82(\mathrm{~s}, 2 \mathrm{H}), 1.71(\mathrm{t}, J=8.0$ $\mathrm{Hz}, 4 \mathrm{H}), 1.63-1.53(\mathrm{~m}, 8 \mathrm{H}), 1.26-1.20(\mathrm{~m}, 4 \mathrm{H}) ;{ }^{13} \mathrm{C} \mathrm{NMR}\left(100 \mathrm{MHz}, \mathrm{CDCl}_{3}\right) \delta: 83.0$, 69.2, 68.3, 39.7, 25.0, 23.1. LRMS (EI, $20 \mathrm{eV}) \mathrm{m} / z$ (\%): $246\left(\mathrm{M}^{+}, 1\right), 210$ (100), 167 (32), 141 (19), 77 (10).

\section{2,4,9,11-Tetramethyl dodeca-5,7-diyne-4,9-diol (2k)}

${ }^{1} \mathrm{H}$ NMR (400 MHz, $\left.\mathrm{CDCl}_{3}\right)$ \&: $4.82(\mathrm{~s}, 2 \mathrm{H}), 1.95-1.56(\mathrm{~m}, 2 \mathrm{H}), 1.61(\mathrm{~d}, J=6.4$ $\mathrm{Hz}, 4 \mathrm{H}), 1.51(\mathrm{~s}, 6 \mathrm{H}), 1.02(\mathrm{~d}, J=5.2 \mathrm{~Hz}, 6 \mathrm{H}), 1.00(\mathrm{~d}, J=5.2 \mathrm{~Hz}, 6 \mathrm{H}) ;{ }^{13} \mathrm{C} \mathrm{NMR}$ $\left(100 \mathrm{MHz}, \mathrm{CDCl}_{3}\right) \delta: 83.4,68.6,67.7,51.5,34.6,25.1,24.2,24.0$. LRMS (EI, $\left.20 \mathrm{eV}\right)$ $m / z(\%): 250\left(\mathrm{M}^{+}, 1\right), 232(1), 85(51), 71(100), 57$ (96), 41 (60).

\section{Acetic acid 6-acetoxy hexa-2,4-diynyl ester (2l)}

${ }^{1} \mathrm{H}$ NMR (400 MHz, $\left.\mathrm{CDCl}_{3}\right) \delta: 4.73$ (s, 4H), $2.10(\mathrm{~s}, 6 \mathrm{H}) ;{ }^{13} \mathrm{C} \mathrm{NMR}(100 \mathrm{MHz}$ $\left.\mathrm{CDCl}_{3}\right) \delta: 169.4,73.6,70.3,52.8,20.6 . \mathrm{LRMS}(\mathrm{EI}, 20 \mathrm{eV}) \mathrm{m} / z(\%): 194\left(\mathrm{M}^{+}, 5\right), 135$ (3), $76(100)$.

\section{1-(2-(4-nitrophenyl)ethynyl)benzene (4)}

H NMR (300 MHz, $\left.\mathrm{CDCl}_{3}\right) \delta: 8.21(\mathrm{~d}, J=8.8 \mathrm{~Hz}, 2 \mathrm{H}), 7.65(\mathrm{~d}, J=8.8 \mathrm{~Hz}, 2 \mathrm{H})$,

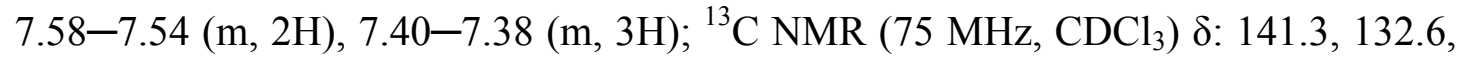
$132.2,130.6,129.6,128.8,124.0,122.5,95.0,87.9$. 


\section{1-(dec-1-ynyl)-4-nitrobenzene (5)}

${ }^{1} \mathrm{H}$ NMR $(400 \mathrm{MHz}) \delta: 8.15(\mathrm{~d}, J=8.8 \mathrm{~Hz}, 2 \mathrm{H}), 7.51(\mathrm{~d}, J=8.8 \mathrm{~Hz}, 2 \mathrm{H}), 2.44(\mathrm{t}, J=$ $7.2 \mathrm{~Hz}, 2 \mathrm{H}), 1.74-1.59(\mathrm{~m}, 2 \mathrm{H}), 1.47-1.43(\mathrm{~m}, 2 \mathrm{H}), 1.31-1.26(\mathrm{~m}, 8 \mathrm{H}), 0.89$ (t, $J=$

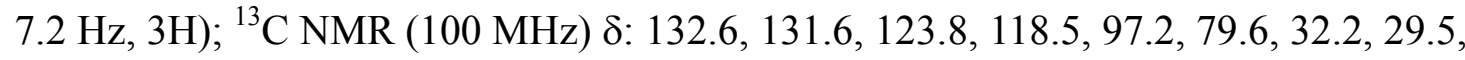
29.4, 29.3, 28.7, 23.0, 19.9, 14.4 .

\section{1-(2-(2-nitrophenyl)ethynyl)benzene (6)}

H NMR (300 MHz, $\left.\mathrm{CDCl}_{3}\right) \delta: 8.10(\mathrm{~d}, J=8.4 \mathrm{~Hz}, 1 \mathrm{H}), 7.73(\mathrm{~d}, J=7.6 \mathrm{~Hz}, 1 \mathrm{H})$, 7.64-7.60 (m, 3H), $7.48(\mathrm{t}, J=8.4 \mathrm{~Hz}, 1 \mathrm{H}), 7.40-7.38(\mathrm{~m}, 3 \mathrm{H}) ;{ }^{13} \mathrm{C}$ NMR $(75 \mathrm{MHz}$, $\left.\mathrm{CDCl}_{3}\right) \delta: 149.5,132.9,132.0,129.2,128.5,128.4,124.7,122.3,118.7,97.1,84.8$.

\section{1,2-diphenylethyne (7)}

${ }^{1} \mathrm{H}$ NMR $\left(300 \mathrm{MHz}, \mathrm{CDCl}_{3}\right) \delta: 7.60-7.51(\mathrm{~m}, 4 \mathrm{H}), 7.39-7.26(\mathrm{~m}, 6 \mathrm{H}) ;{ }^{13} \mathrm{C}$ NMR $(75$ $\left.\mathrm{MHz}, \mathrm{CDCl}_{3}\right) \delta: 132.0,128.7,128.6,123.7,89.7$.

\section{1-(2-p-tolylethynyl)benzene (8)}

${ }^{1} \mathrm{H}$ NMR $(300 \mathrm{MHz}) \delta: 7.54-7.50(\mathrm{~m}, 2 \mathrm{H}), 7.43(\mathrm{~d}, J=8.1 \mathrm{~Hz}, 2 \mathrm{H}), 7.35-7.31(\mathrm{~m}$, $3 \mathrm{H}), 7.15(\mathrm{~d}, J=7.8 \mathrm{~Hz}, 2 \mathrm{H}), 2.36(\mathrm{~s}, 3 \mathrm{H}) ;{ }^{13} \mathrm{C}$ NMR $(100 \mathrm{MHz}) \delta: 138.7,132.9$, $131.9,129.6,129.5,128.8,128.7,128.4,89.1,83.4,21.9$. 


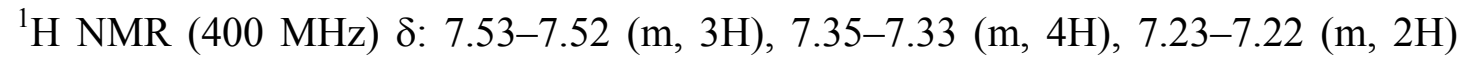

2.52 (s, 3H); ${ }^{13} \mathrm{C}$ NMR (100 MHz) $\delta: 140.2,132.6,131.9,131.6,129.5,129.2,128.4$, $128.3,128.2,125.6,88.4,81.7,20.7$.

\section{1-methoxy-4-(2-phenylethynyl)benzene (10)}

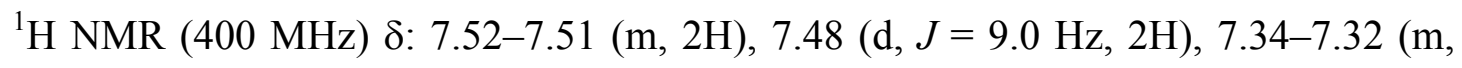
$3 \mathrm{H}), 6.88(\mathrm{~d}, J=8.8 \mathrm{~Hz}, 2 \mathrm{H}), 3.83(\mathrm{~s}, 3 \mathrm{H}) ;{ }^{13} \mathrm{C} \mathrm{NMR}(100 \mathrm{MHz}) \delta: 159.6,133.0$, $131.4,128.3,127.9,123.6,115.4,114.0,89.4,88.1,55.3$.

\section{(C) References:}

(1) Lei, A.; Srivastava, M.; Zhang, X. J. Org. Chem. 2002, 67, 1969 and references cited therein.

(2) Chow, H.-F.; Wan, C.-W.; Low, K.-H.; Yeung, Y.-Y. J. Org. Chem. 2001, 66, 1910.

(3) Vlassa, M; Ciocan-Tarta, I.; Margineanu, F.; Oprean, I. Tetrahedron, 1996, 52, 1337.

(4) Nishimura, T.; Araki, H.; Maeda, Y.; Uemura S. Org. Lett. 2003, 5, 2997.

(5) Elangovan, A.; Wang, Y.-H.; Ho, T.-I. Org. Lett. 2003, 5, 1841.

(6) Feuerstein, M.; Berthiol, F.; Doucet, H.; Santelli, M. Synthesis 2004, 1281.

(7) Wolf, C.; Lerebours, R. Org. Biomol. Chem. 2004, 2, 2161.

(8) Hierso, J.-C.; Fihri, A.; Amardeil, R.; Meunier, P.; Doucet, H.; Santelli, M.; Ivanov, V. V. Org. Lett. 2004, 6, 3473.

(9) Heuzé, K.; Méry, D.; Gauss, D.; Blais, J.-C.; Astruc, D. Chem. Eur. J. 2004, 10, 3936. 
(10) Cheng, J.; Sun, Y.; Wang, F.; Guo, M.; Xu, J.-H.; Pan, Y.; Zhang, Z. J. Org. Chem. 2004, 69, 5428.

(12) Urgaonkar, S.; Verkade, J. G. J. Org. Chem. 2004, 69, 5752.

(13) Feuerstein, M.; Berthiol, F.; Doucet, H.; Santelli, M. Synthesis 2004, 1281. 
(D) NMR spectra

1,4-Diphenyl buta-1,3-diyne (2a)

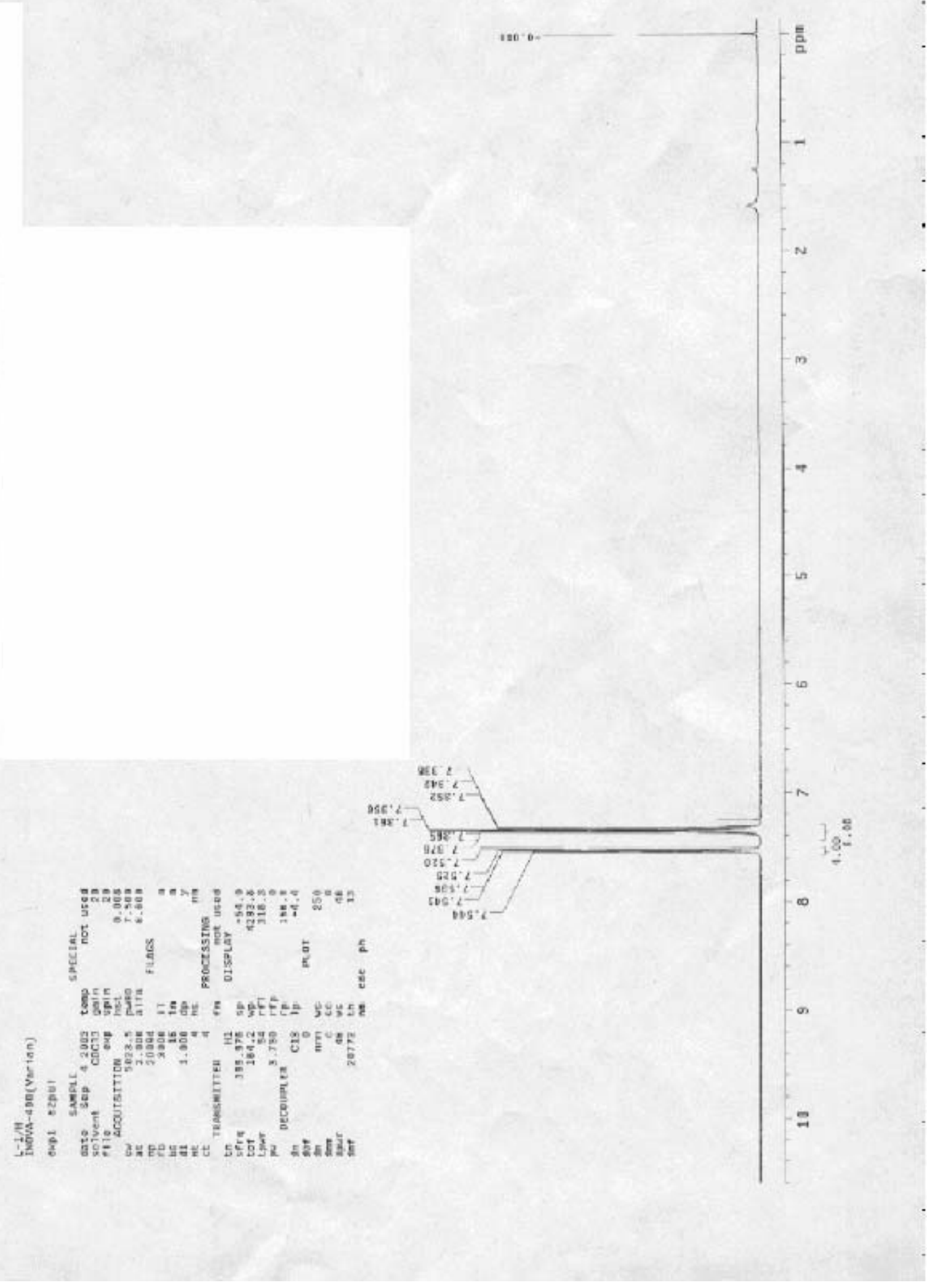


1,4-Diphenyl buta-1,3-diyne (2a)

象

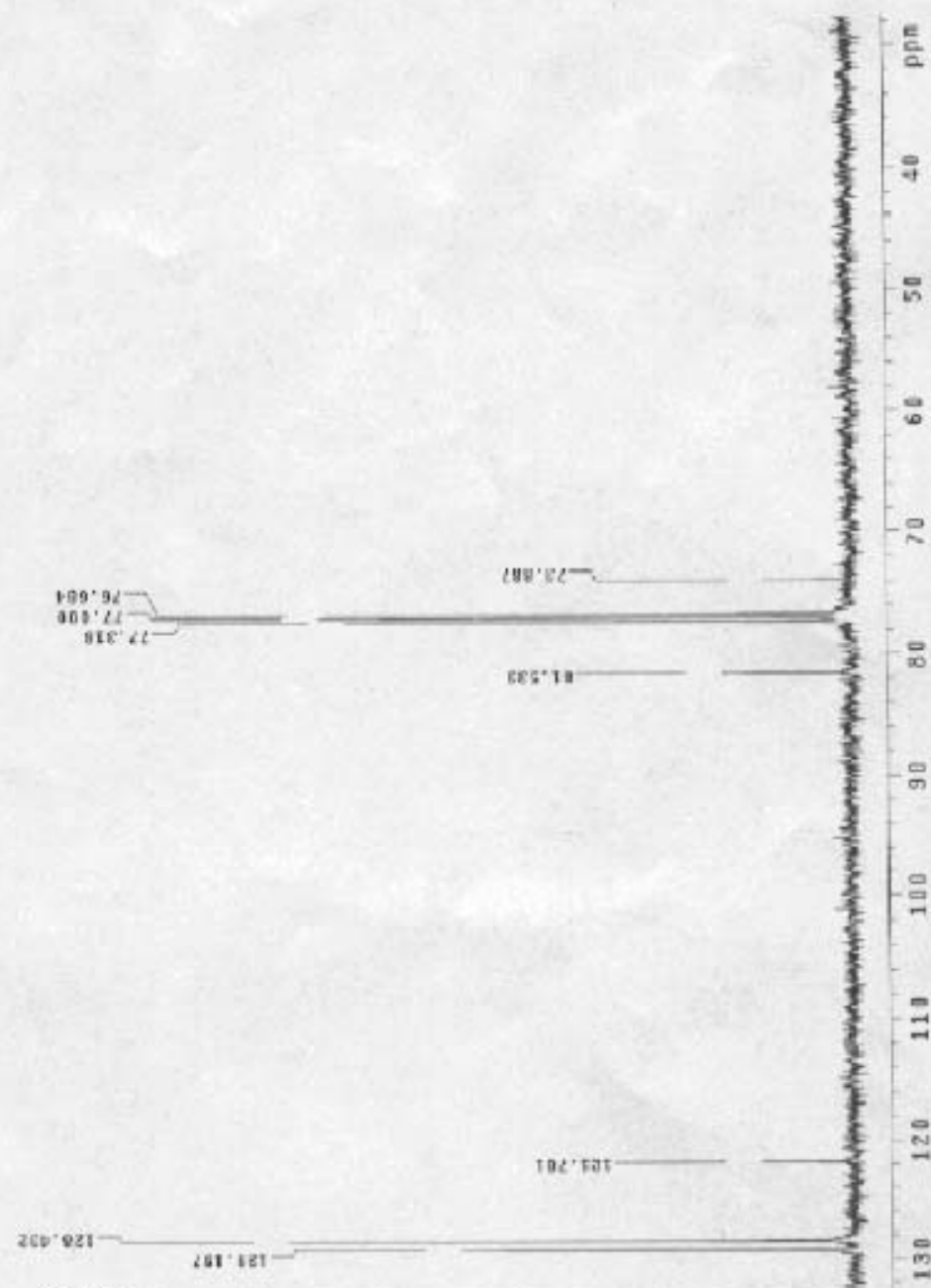

zav' $a \varepsilon_{1}$

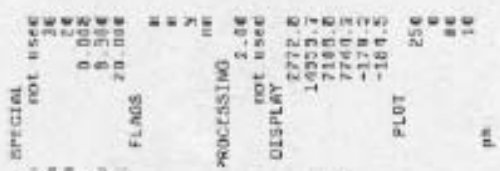

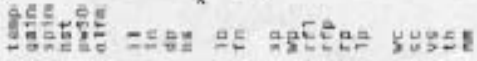

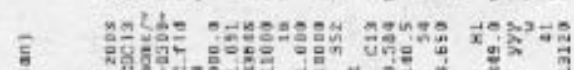

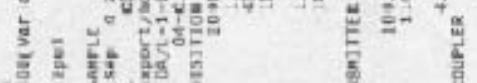

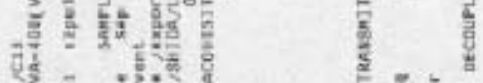

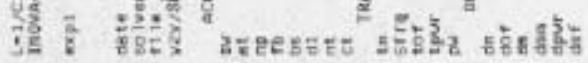

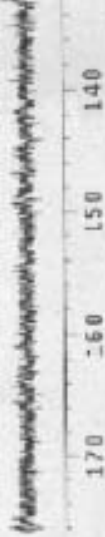


1,4-Bis( $p$-methylphenyl) buta-1,3-diyne (2b)
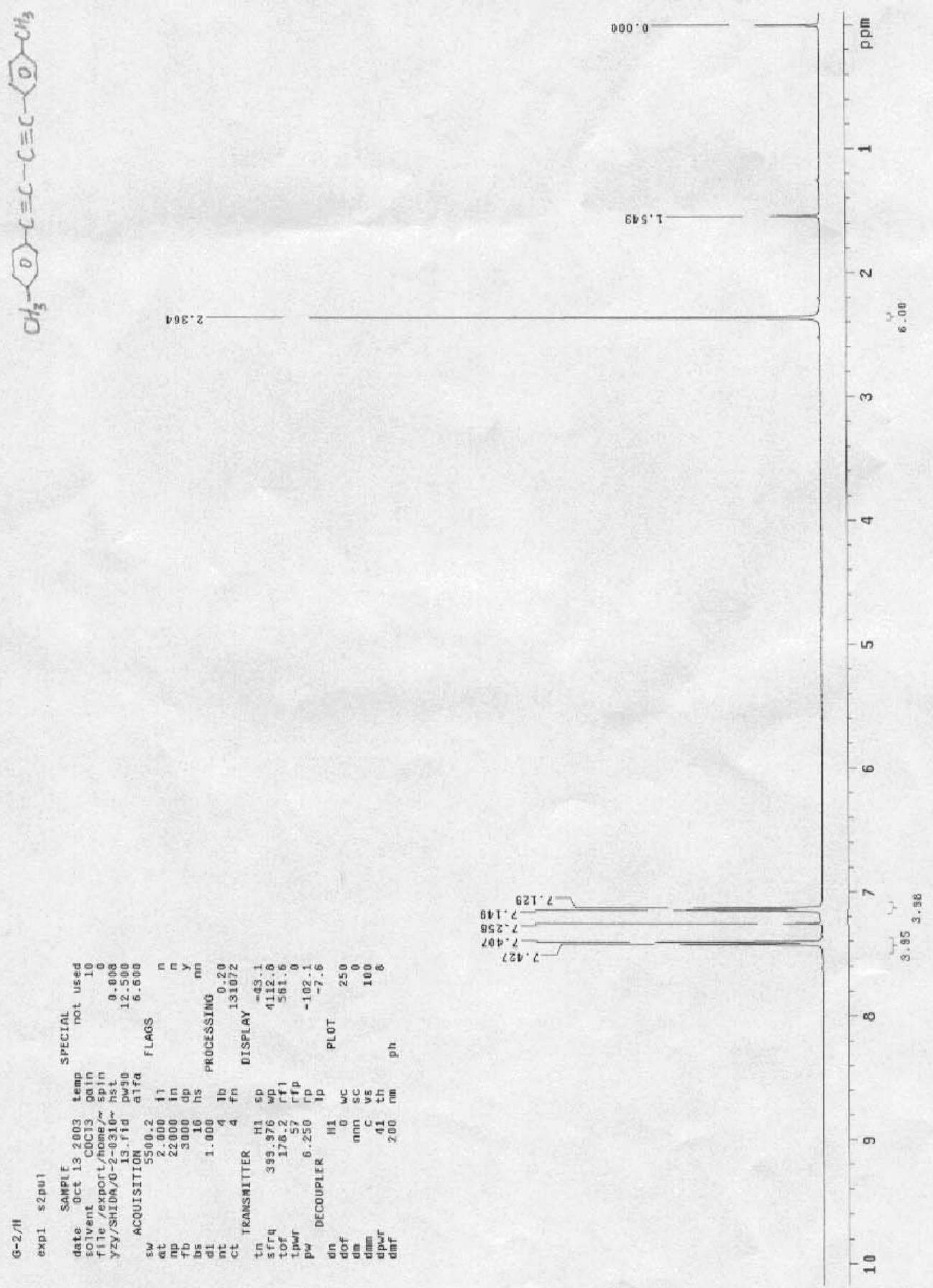
1,4-Bis(p-methylphenyl) buta-1,3-diyne (2b)

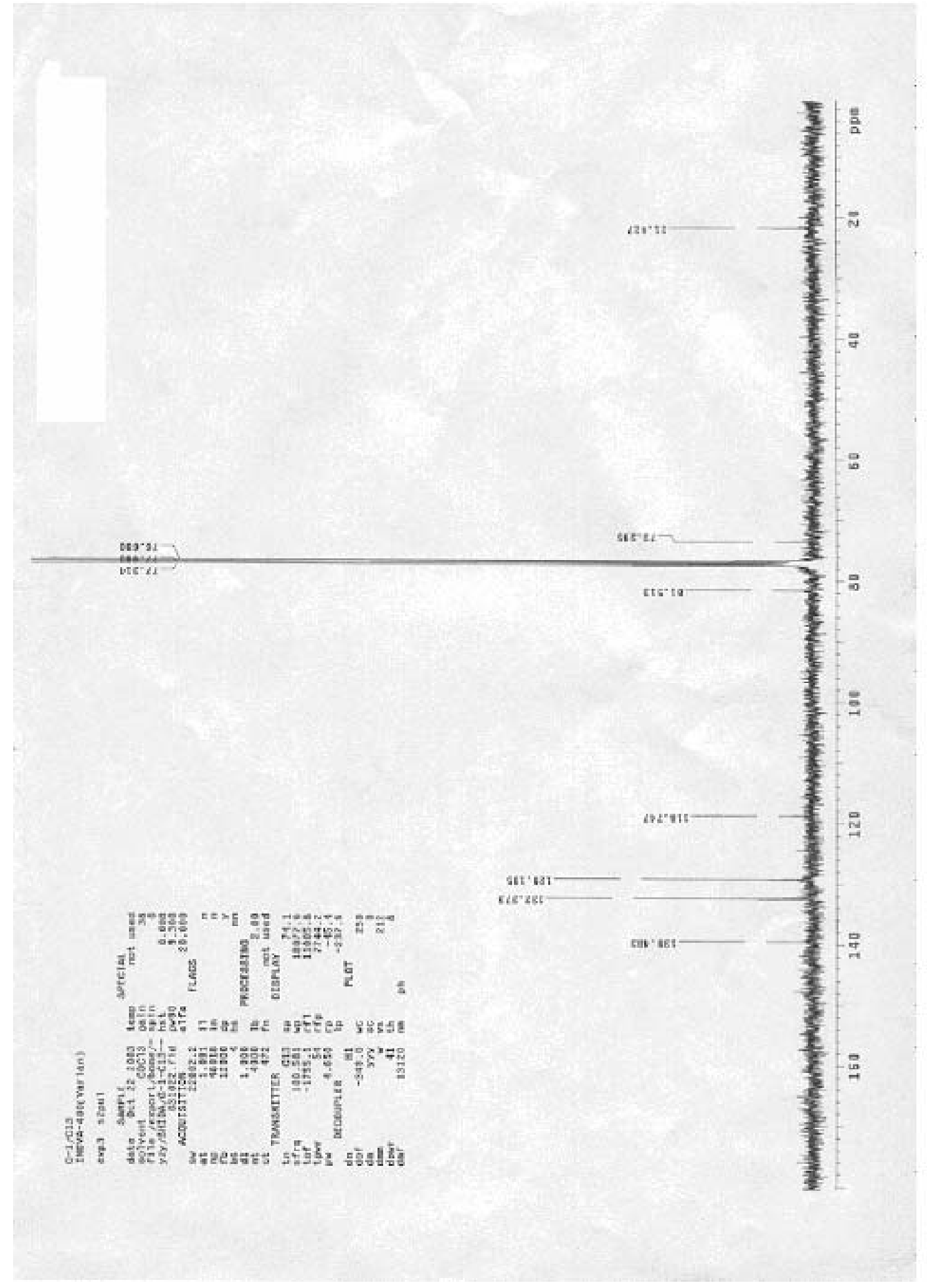


1,4-Bis(p-methoxyphenyl) buta-1,3-diyne (2c)

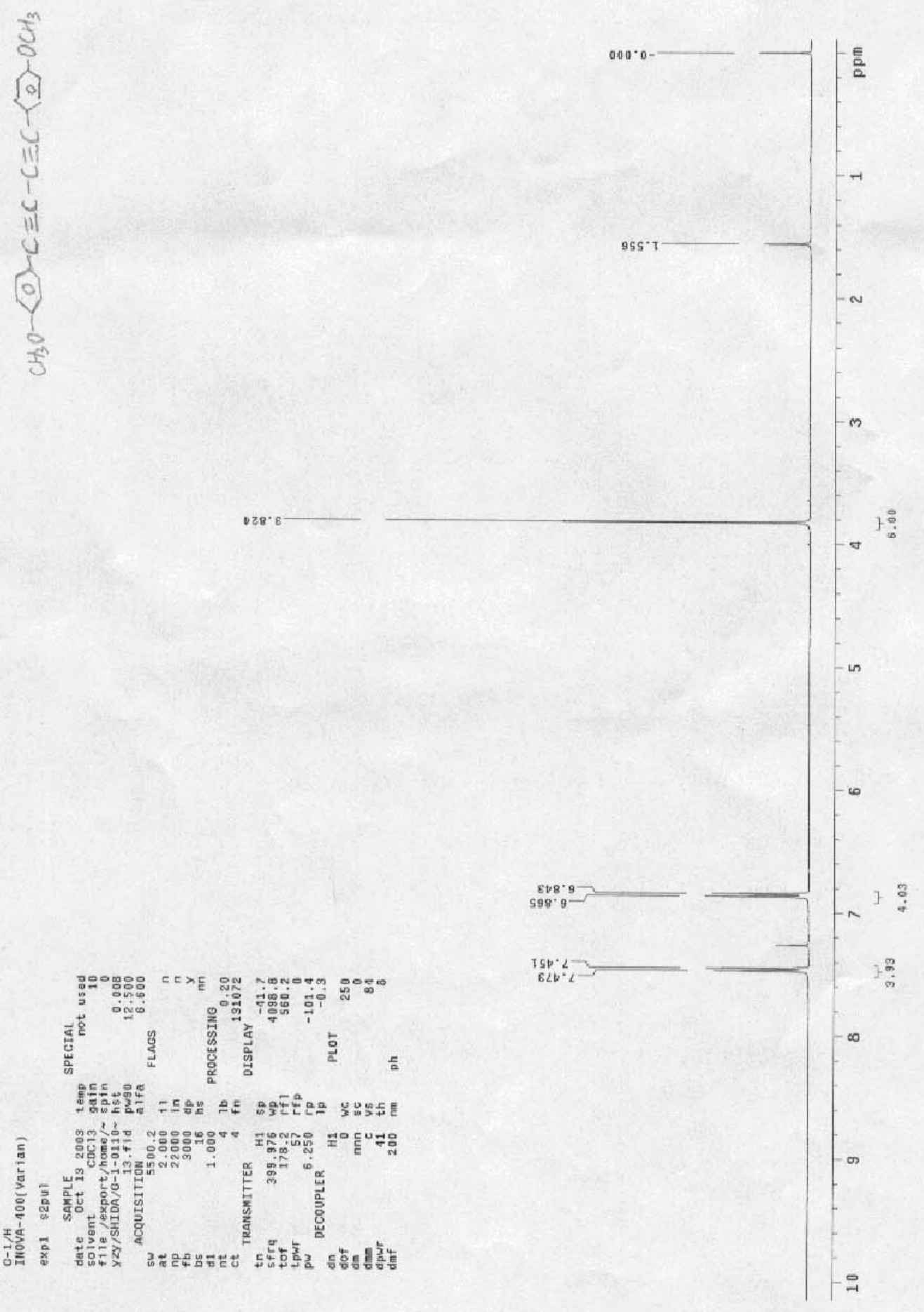


1,4-Bis (p-methoxyphenyl) buta-1,3-diyne (2c)

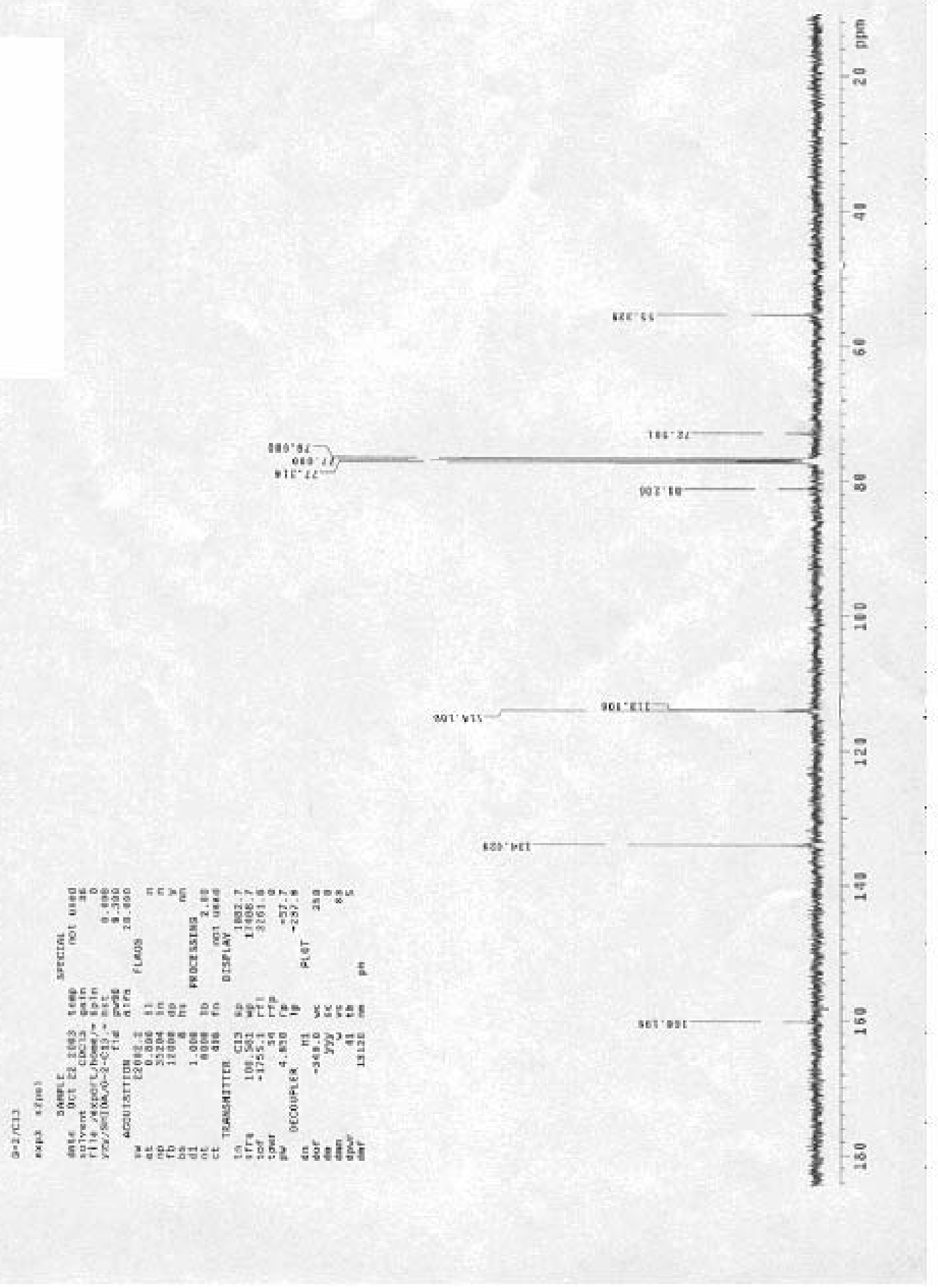


1,4-Bis(trifloromethylphenyl) buta-1,3-diyne (2d)
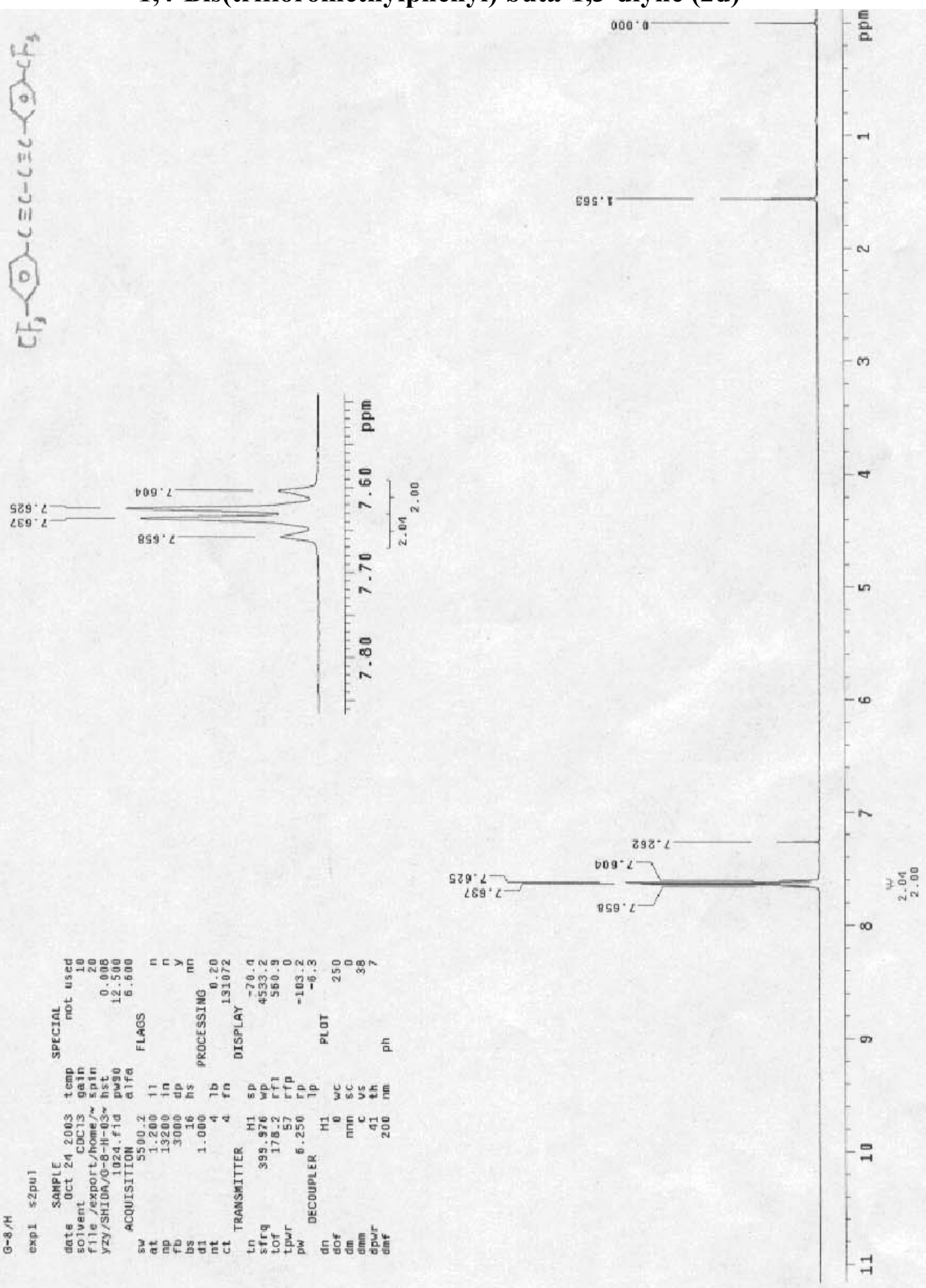
1,4-Bis(trifloromethylphenyl) buta-1,3-diyne (2d)

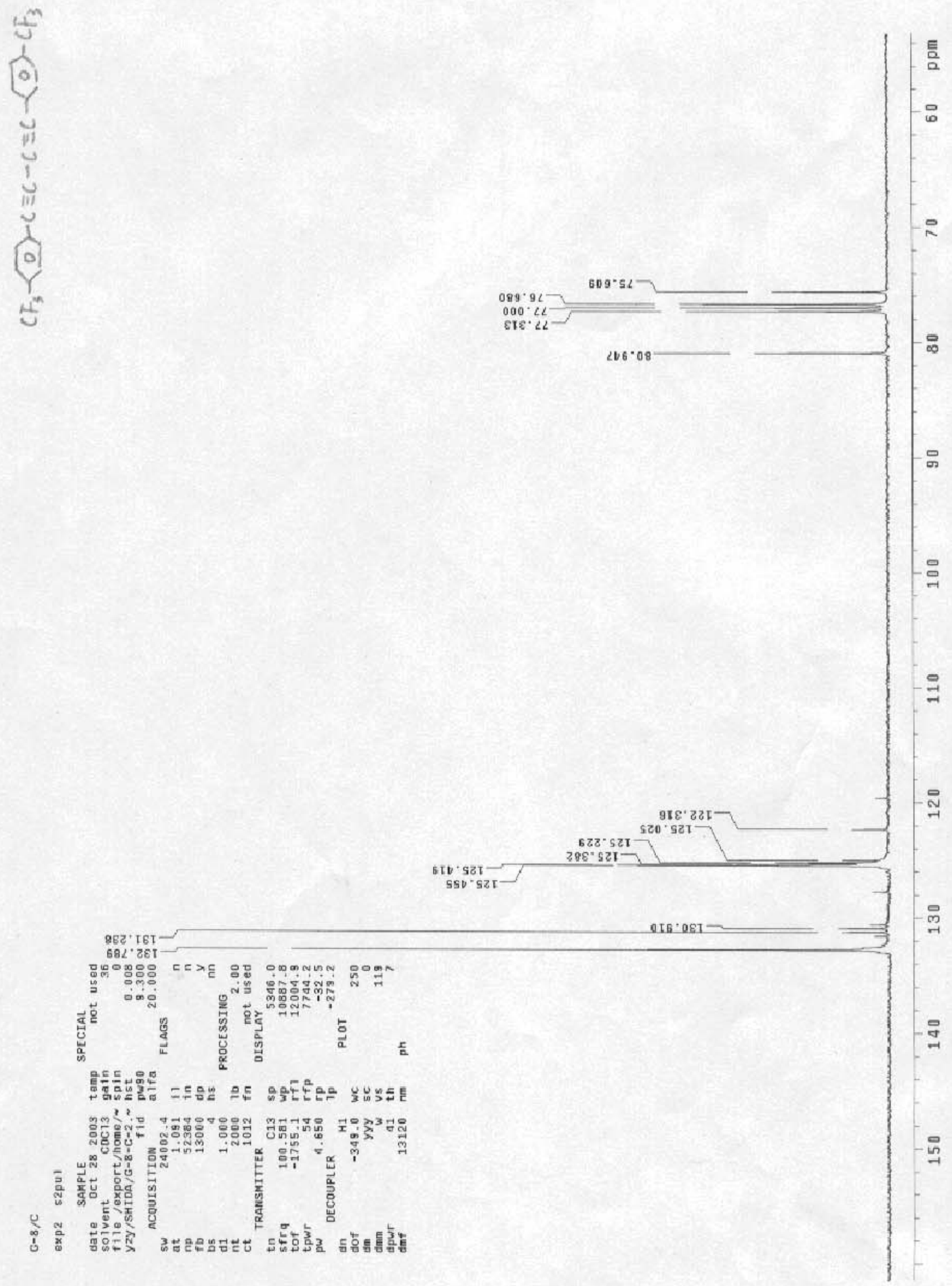


1,4-Bis(2-pyridine)buta-1,3-diyne (2e)

ए3.
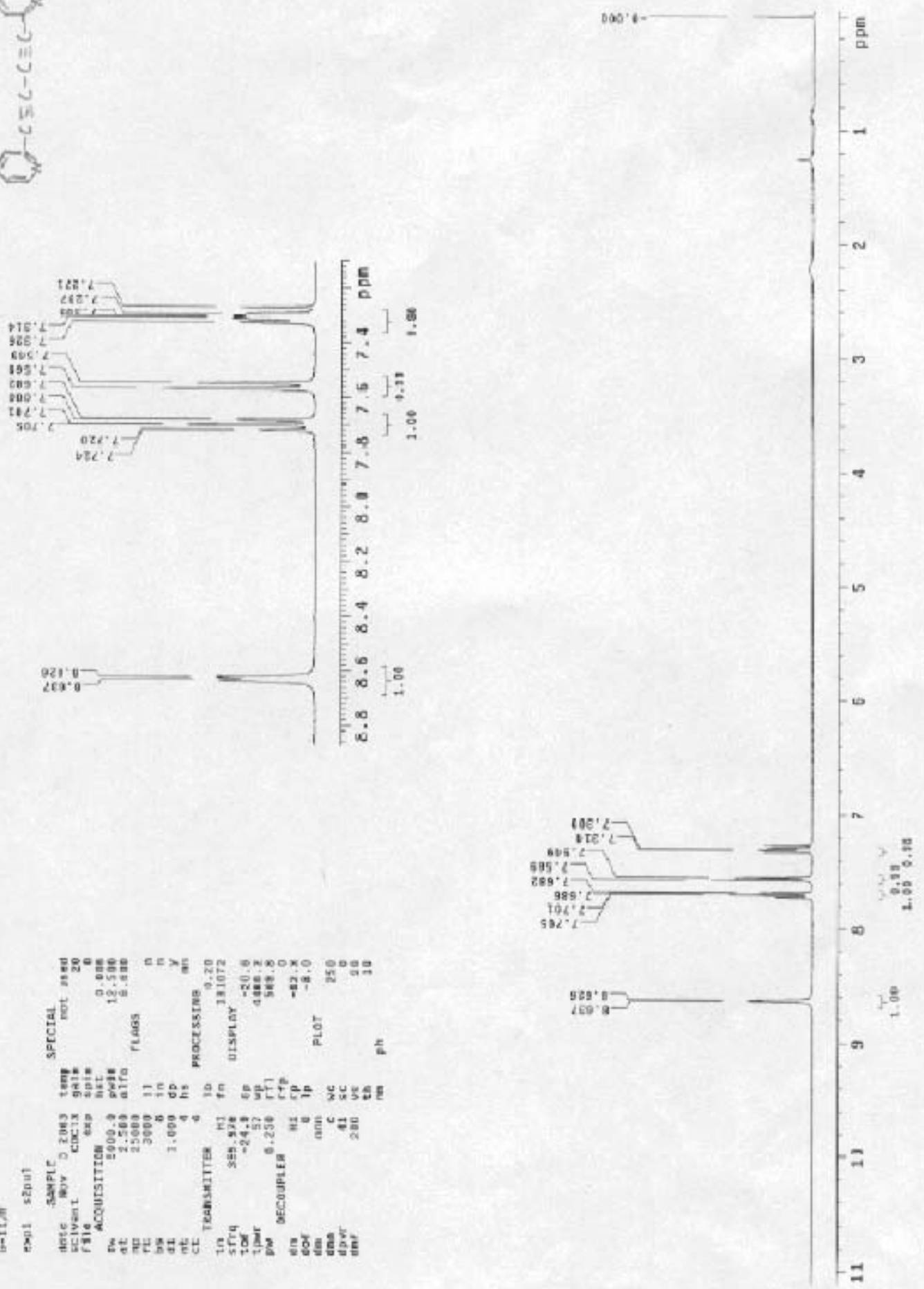
1,4-Bis(2-pyridine)buta-1,3-diyne (2e)

ए3.
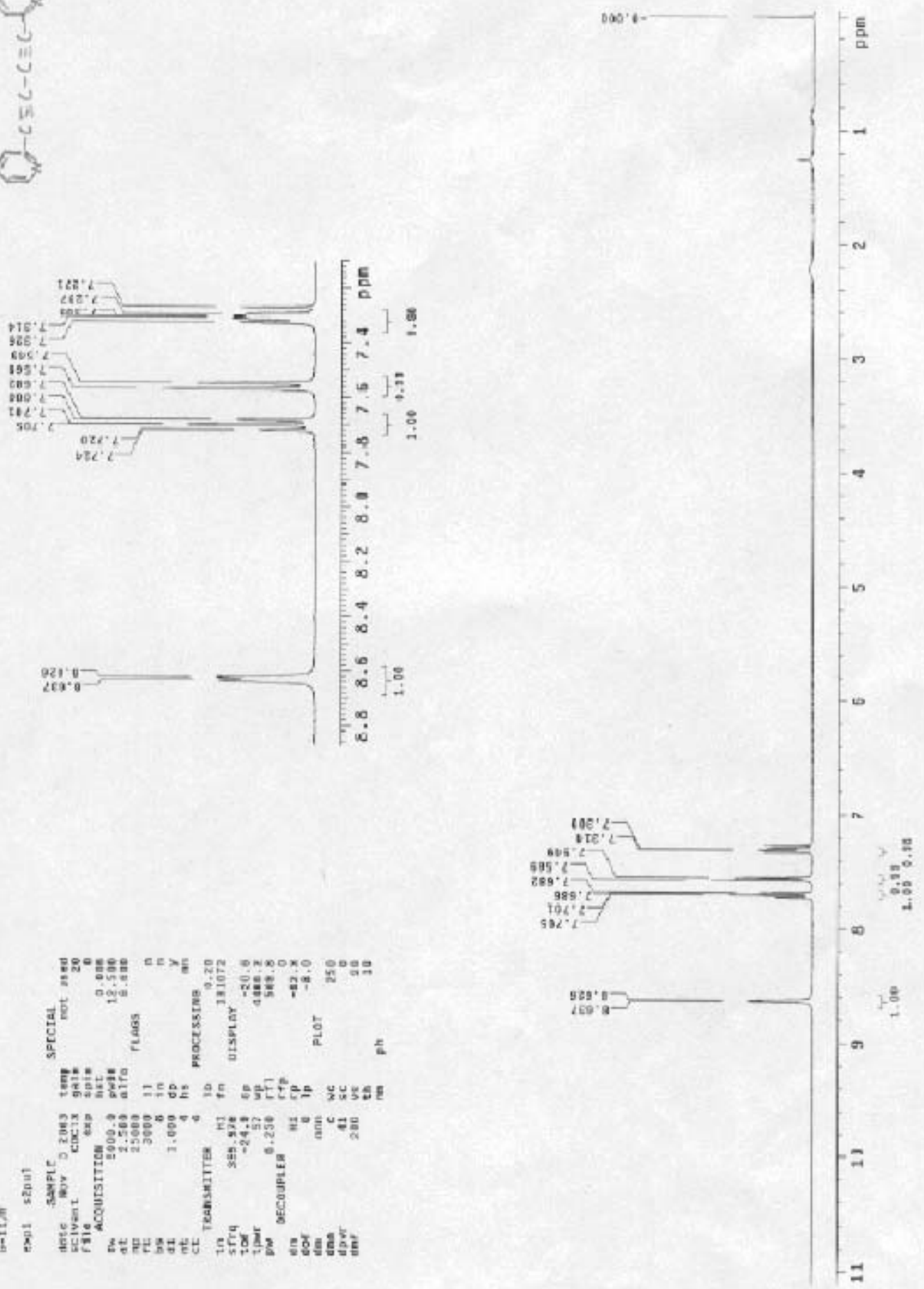
Tetradeca-6,8-diyne (2f)

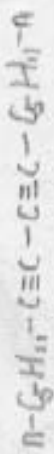
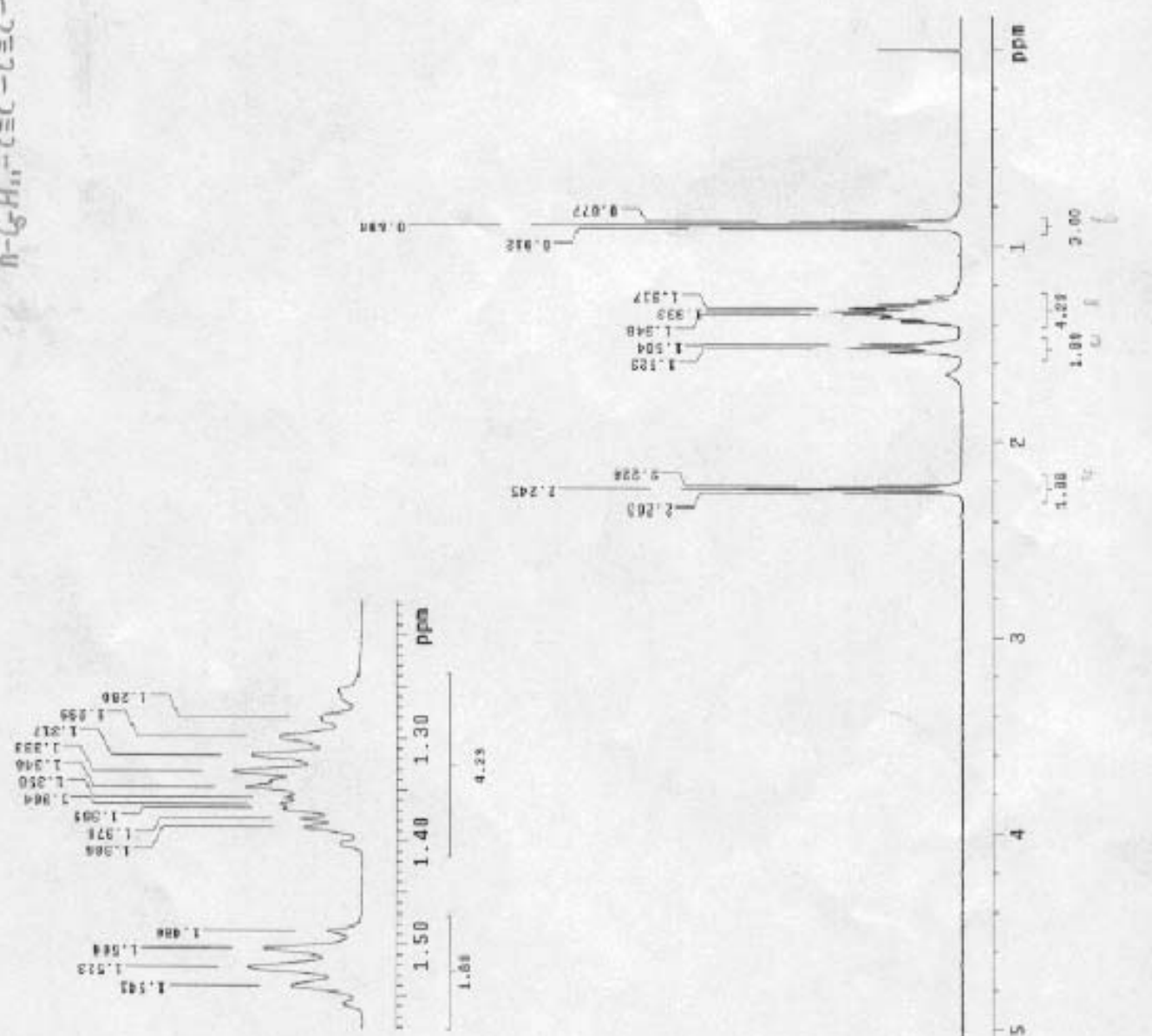

ํำำ

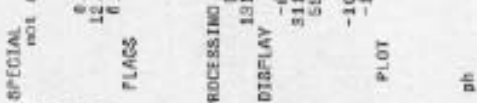

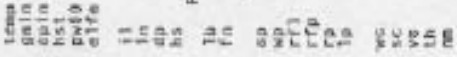

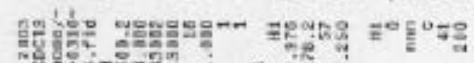

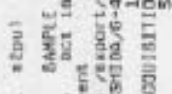

के 
Tetradeca-6,8-diyne (2f)

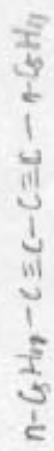

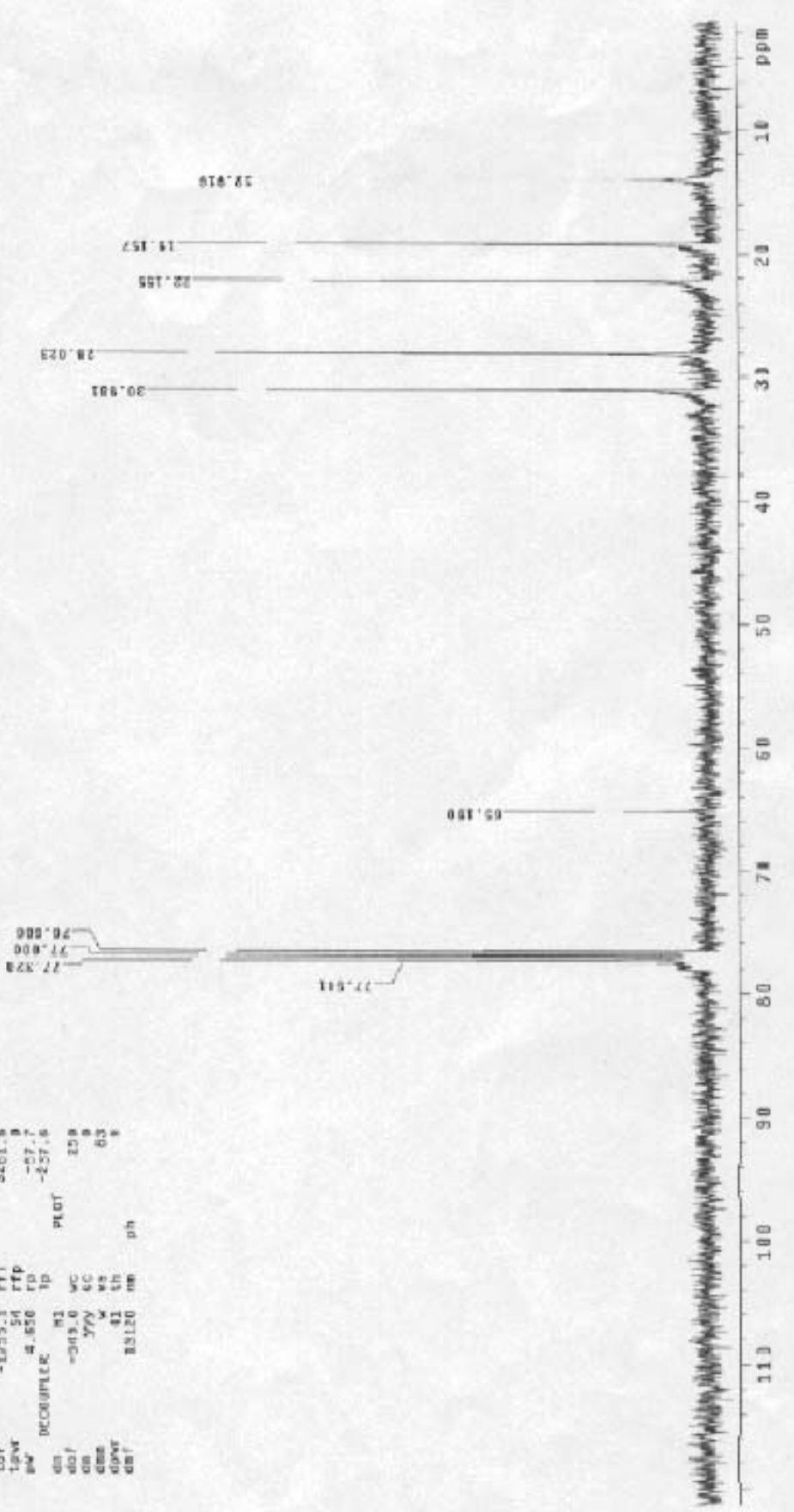




\section{Icosa-9,11-diyne (2g)}

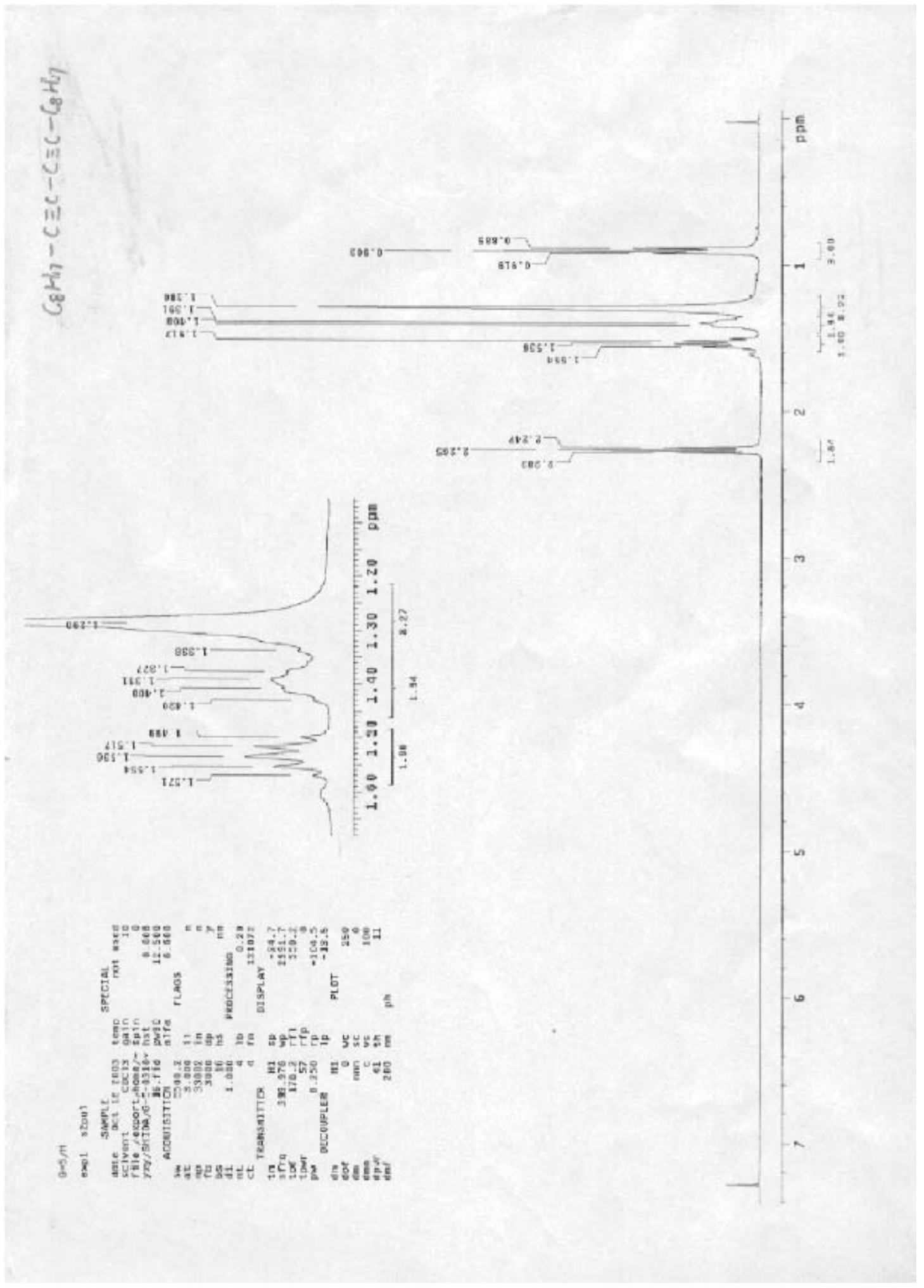




\section{Icosa-9,11-diyne (2g)}

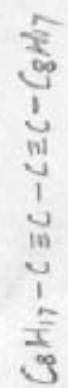

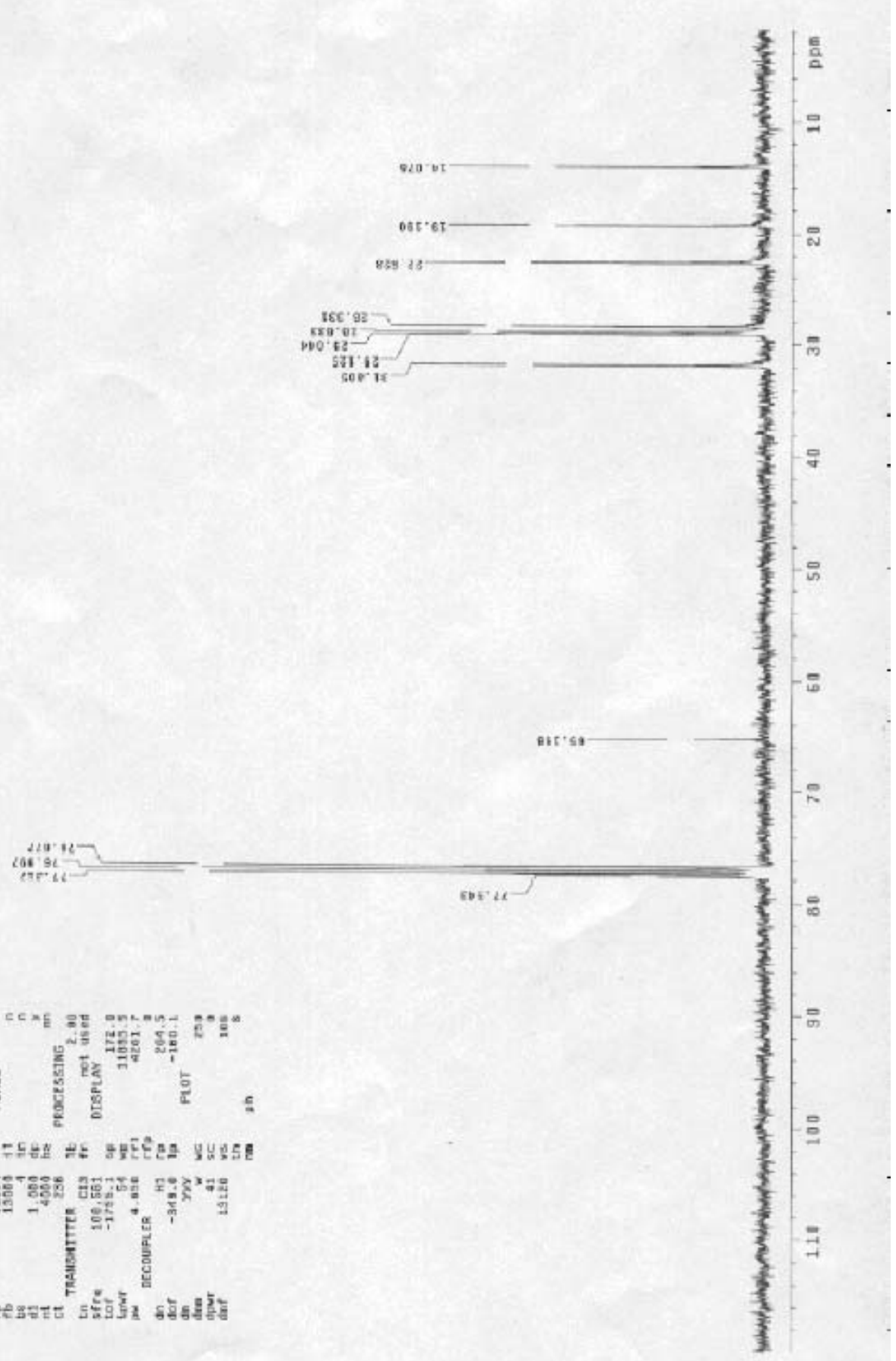


2,2,7,7-Tetramethyl octa-3,5-diyne (2h)

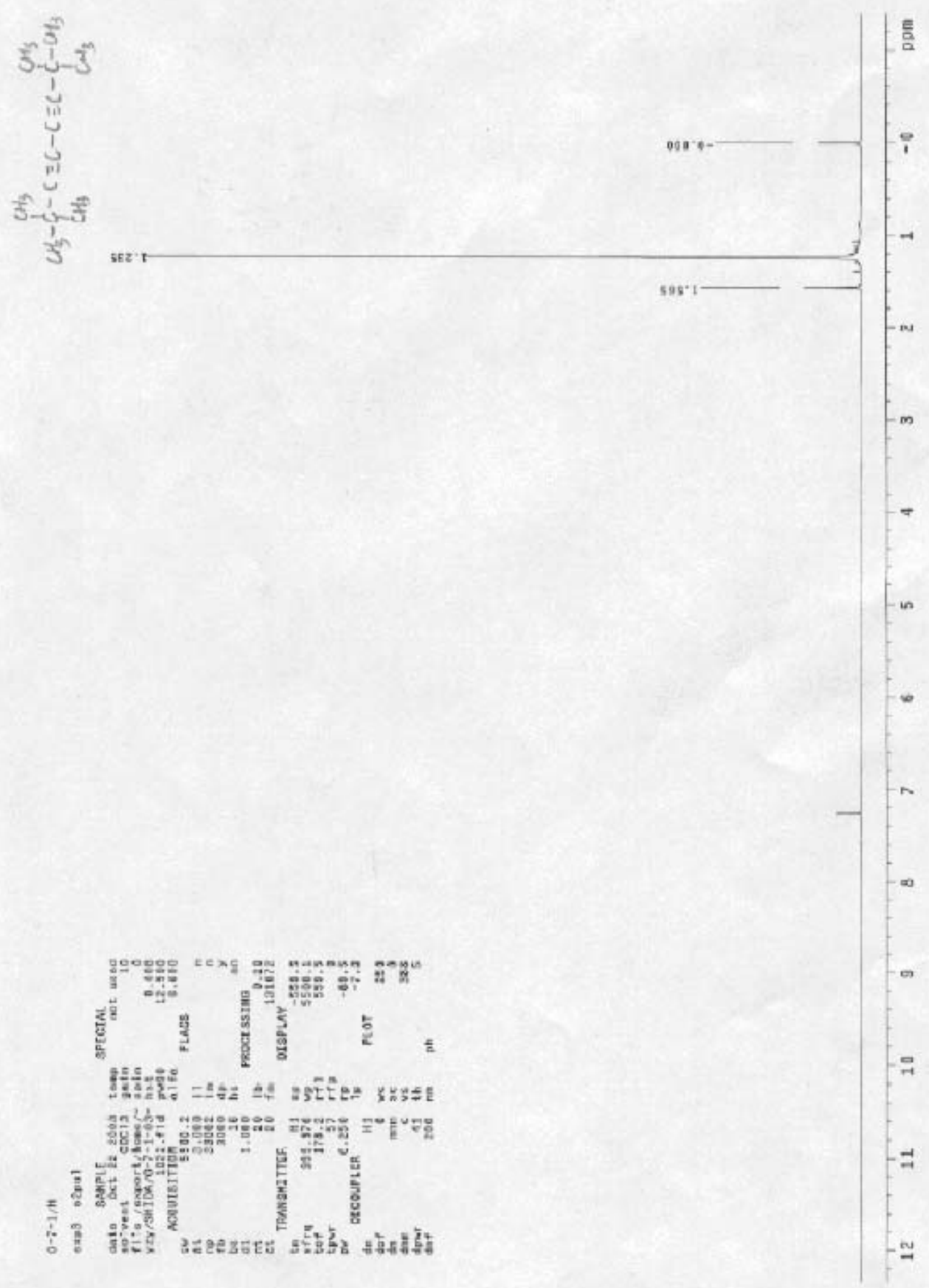


2,2,7,7-Tetramethyl octa-3,5-diyne (2h)

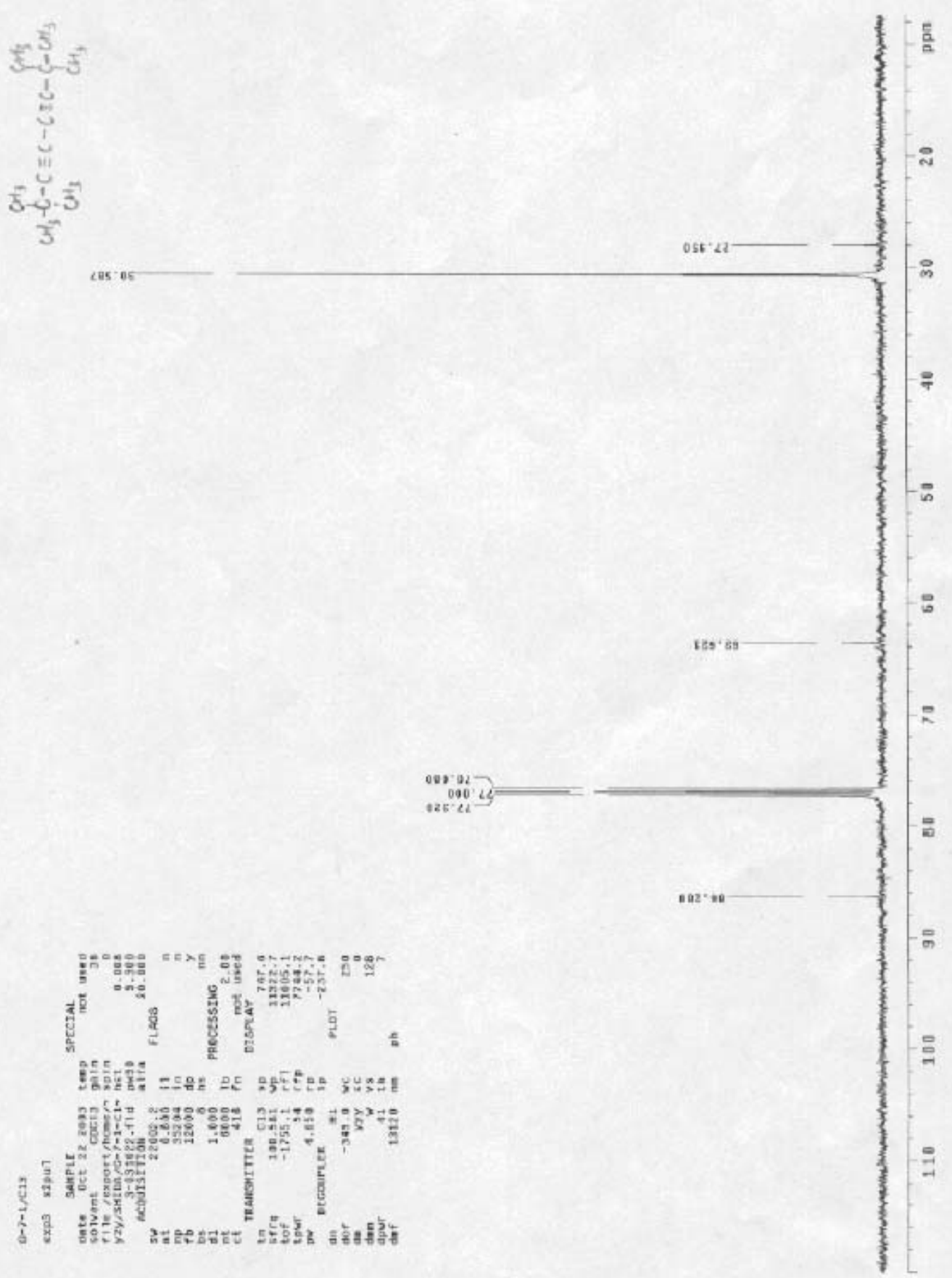


1,4-Bis(cyclohex-1-enyl) buta-1,3-diyne (2i)

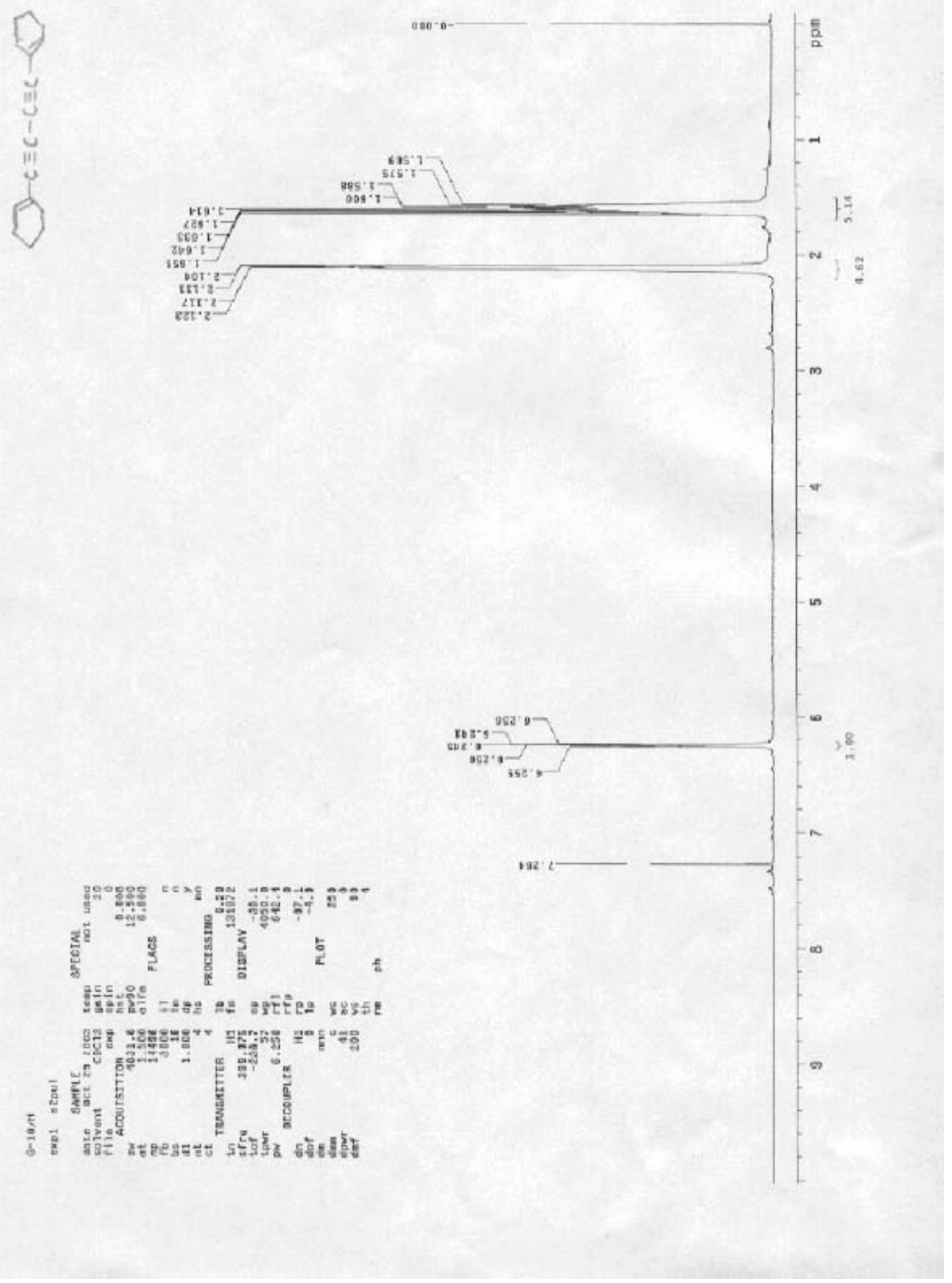


1,4-Bis(cyclohex-1-enyl) buta-1,3-diyne (2i)

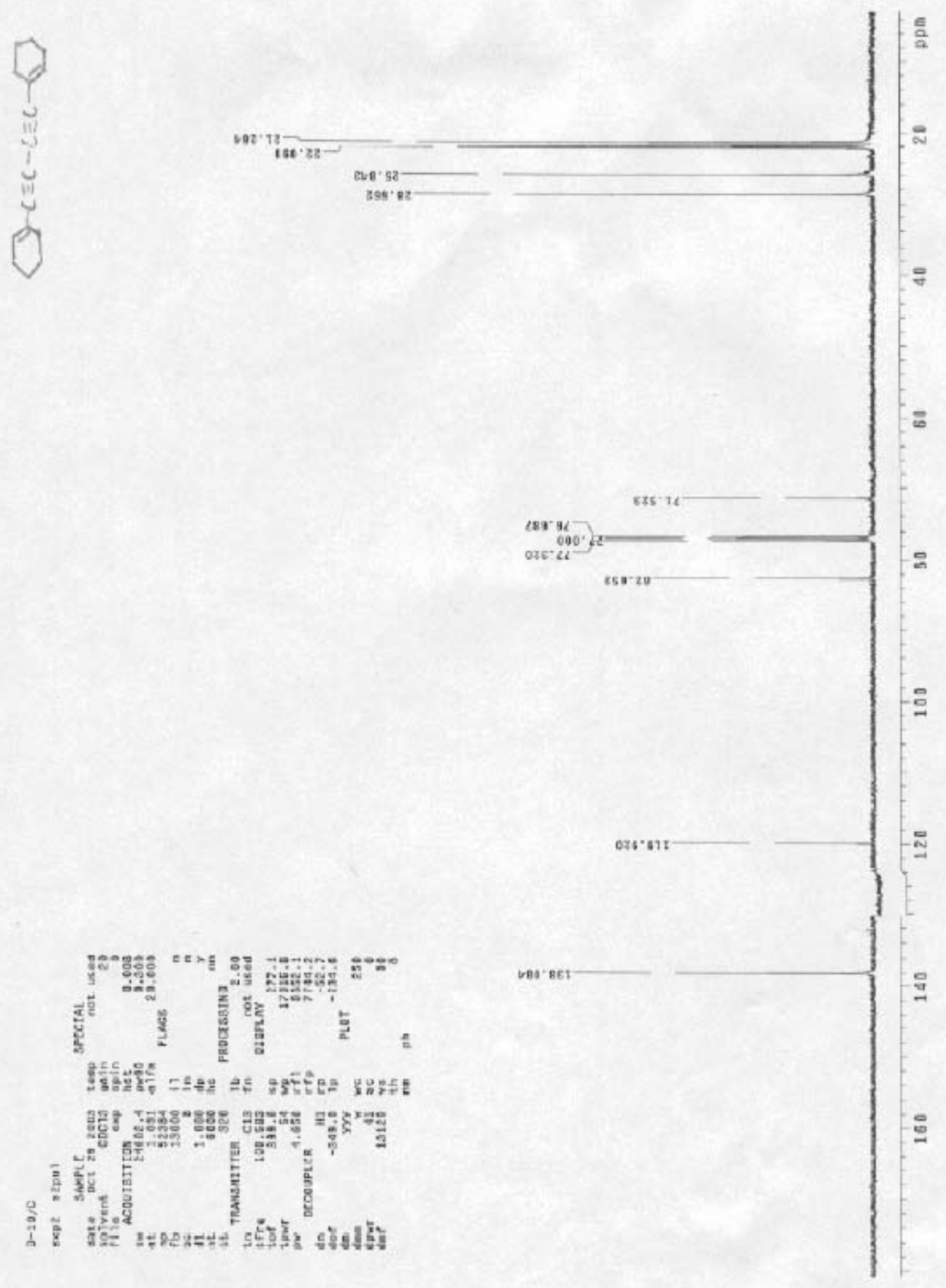


1,4-Bis(1-hydroxycyclohexyl) buta-1,3-diyne (2j)
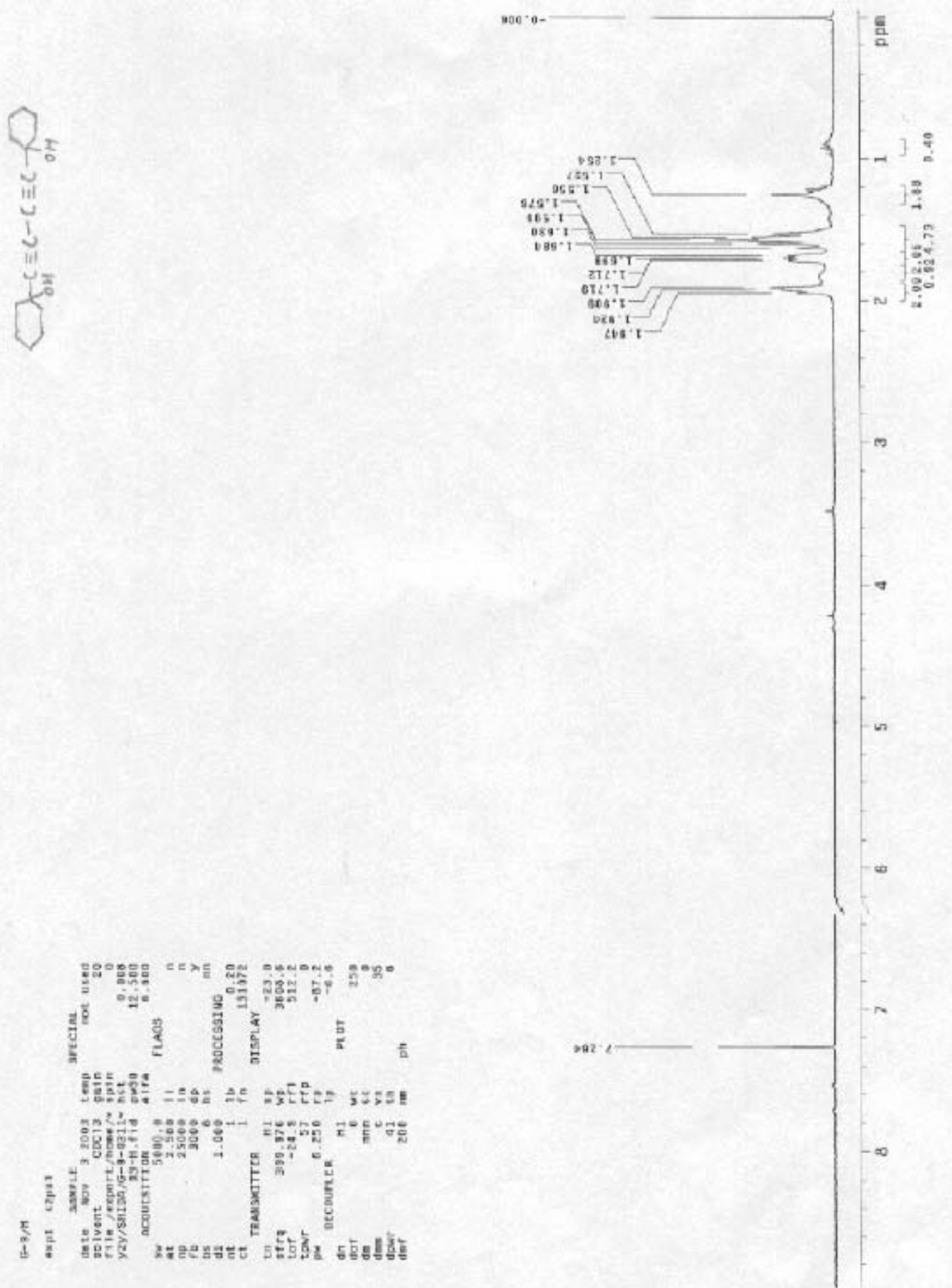
1,4-Bis(1-hydroxycyclohexyl) buta-1,3-diyne (2j)

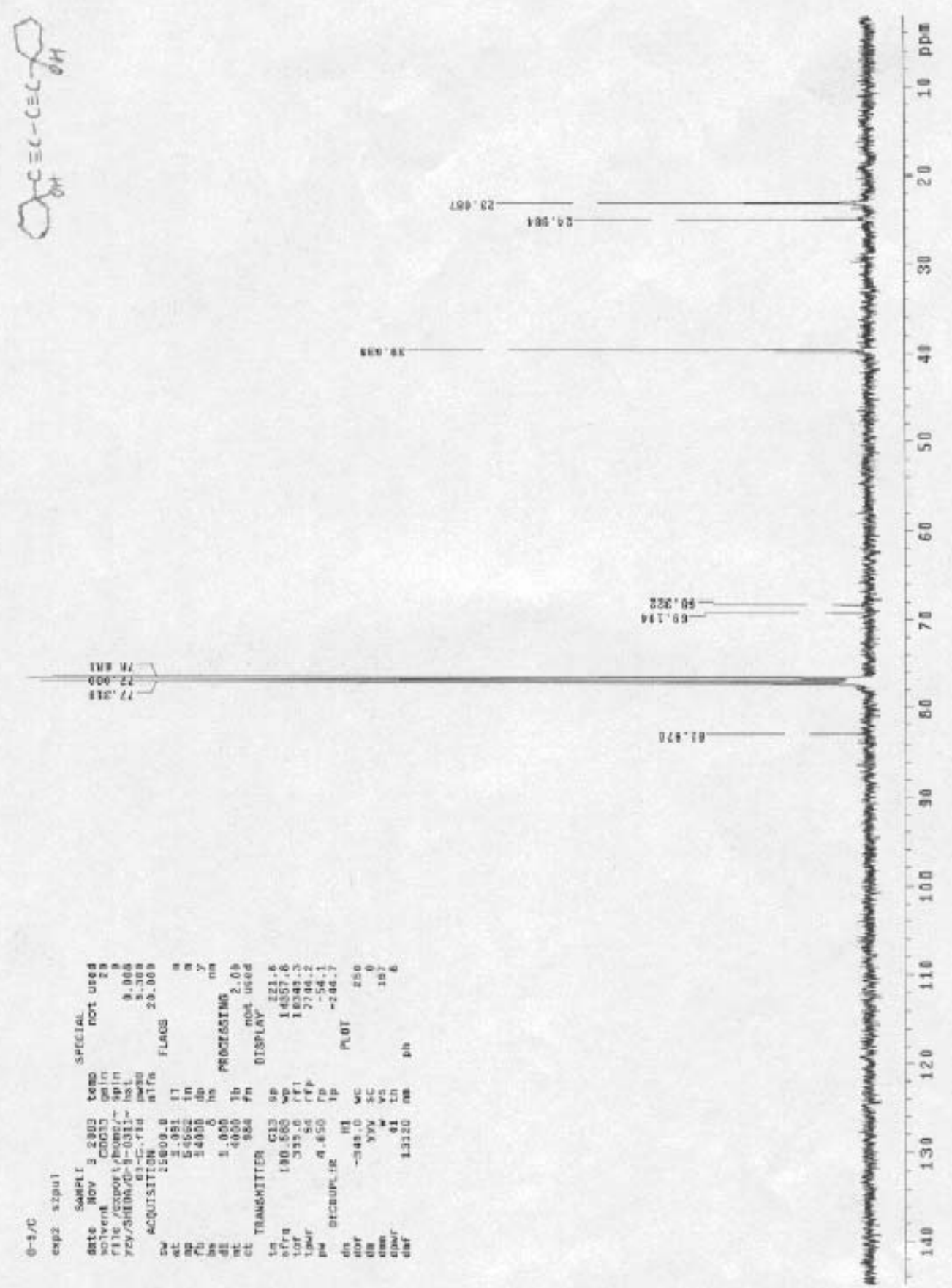




\section{2,4,9,11-Tetramethyl dodeca-5,7-diyne-4,9-diol (2k)}
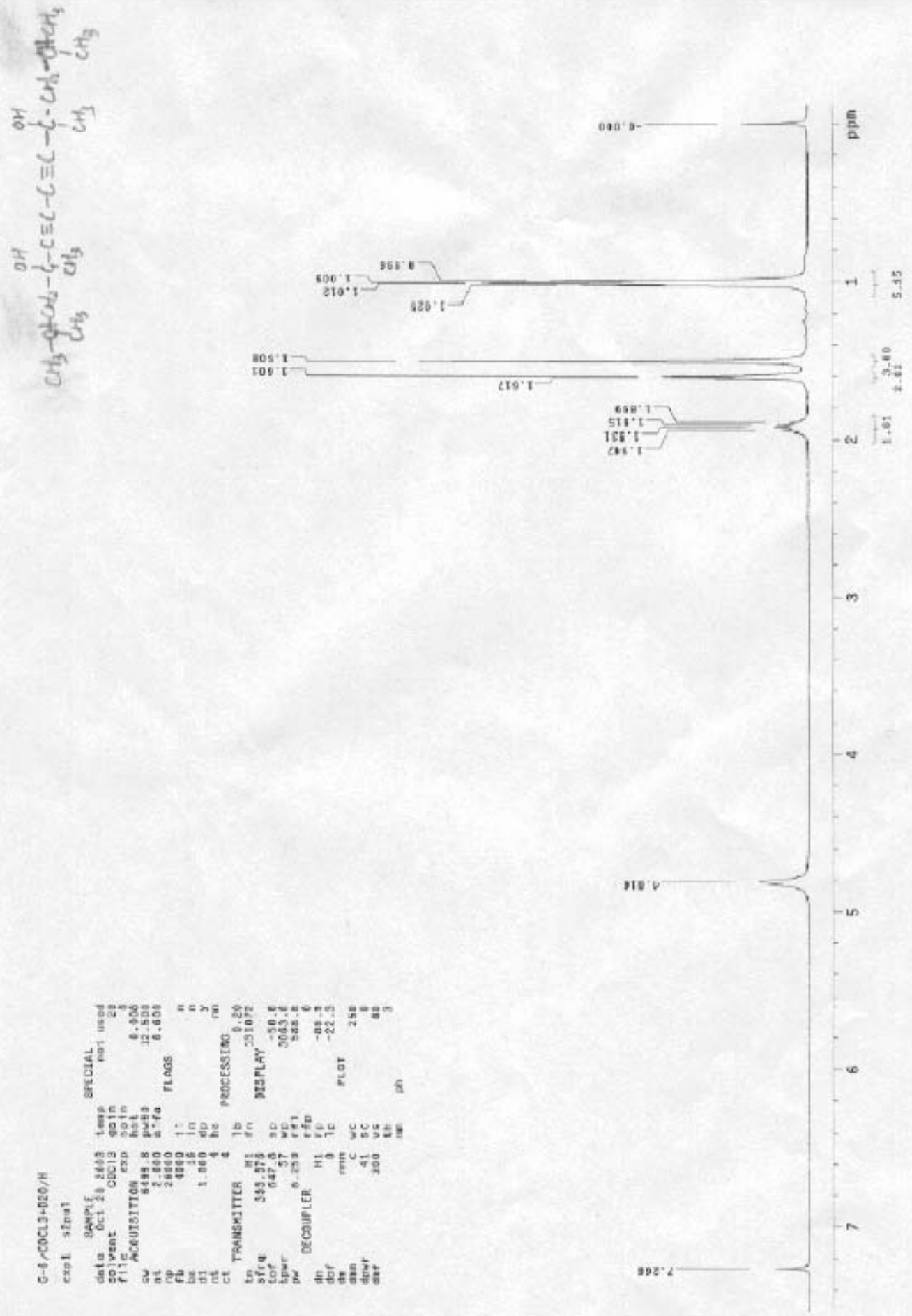
2,4,9,11-Tetramethyl dodeca-5,7-diyne-4,9-diol (2k)

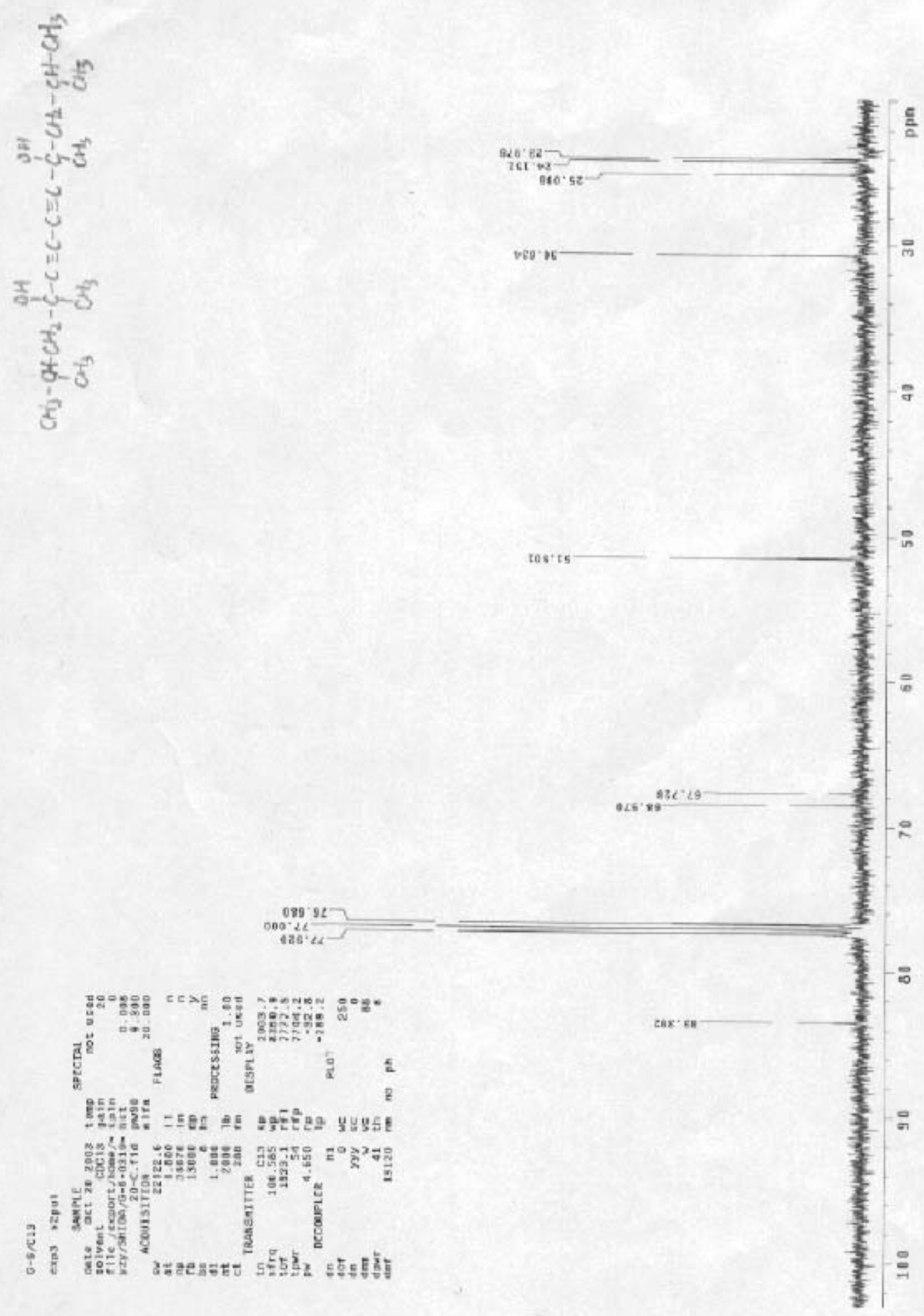


Acetic acid 6-acetoxy hexa-2,4-diynyl ester (2l)

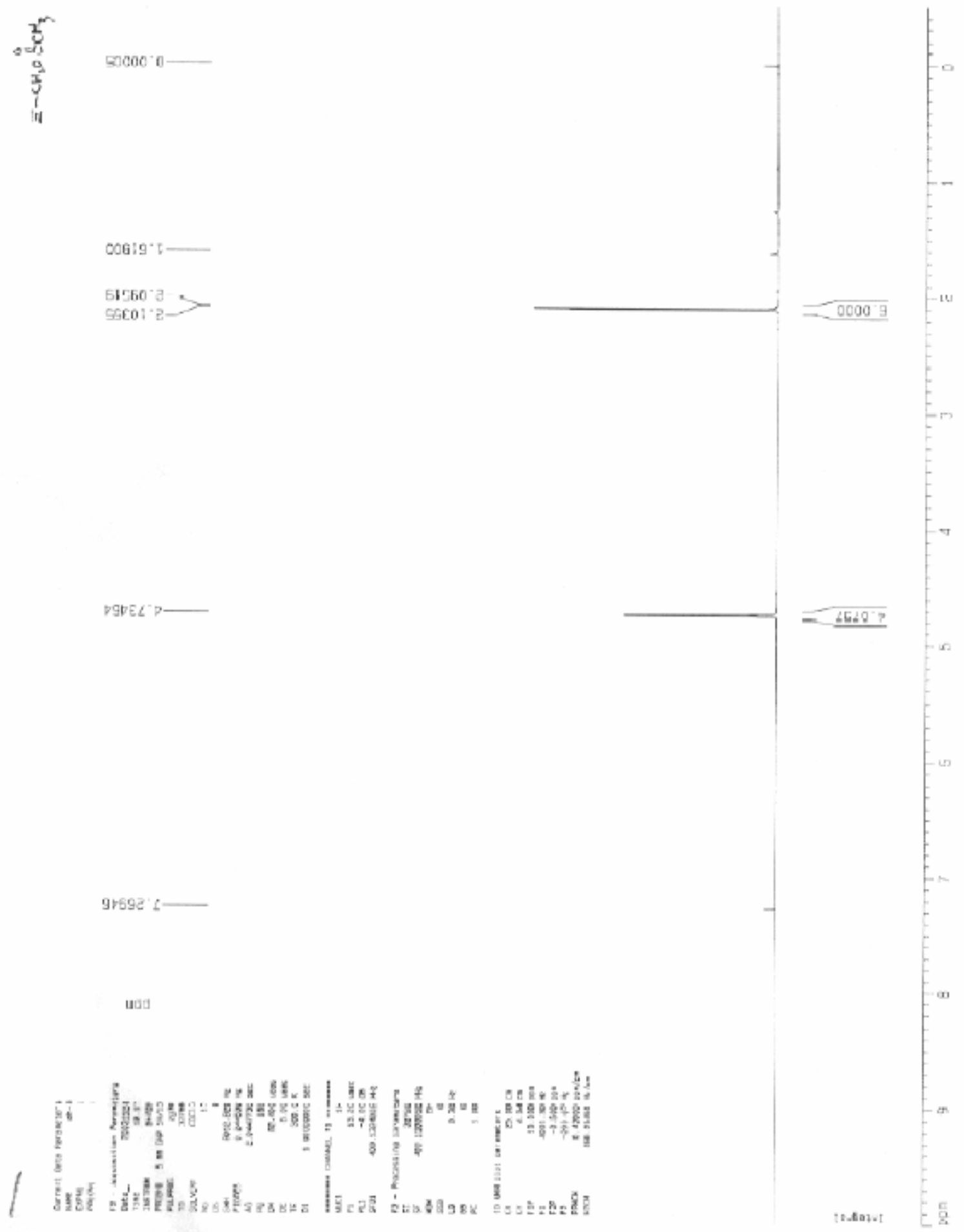


Acetic acid 6-acetoxy hexa-2,4-diynyl ester (2l)

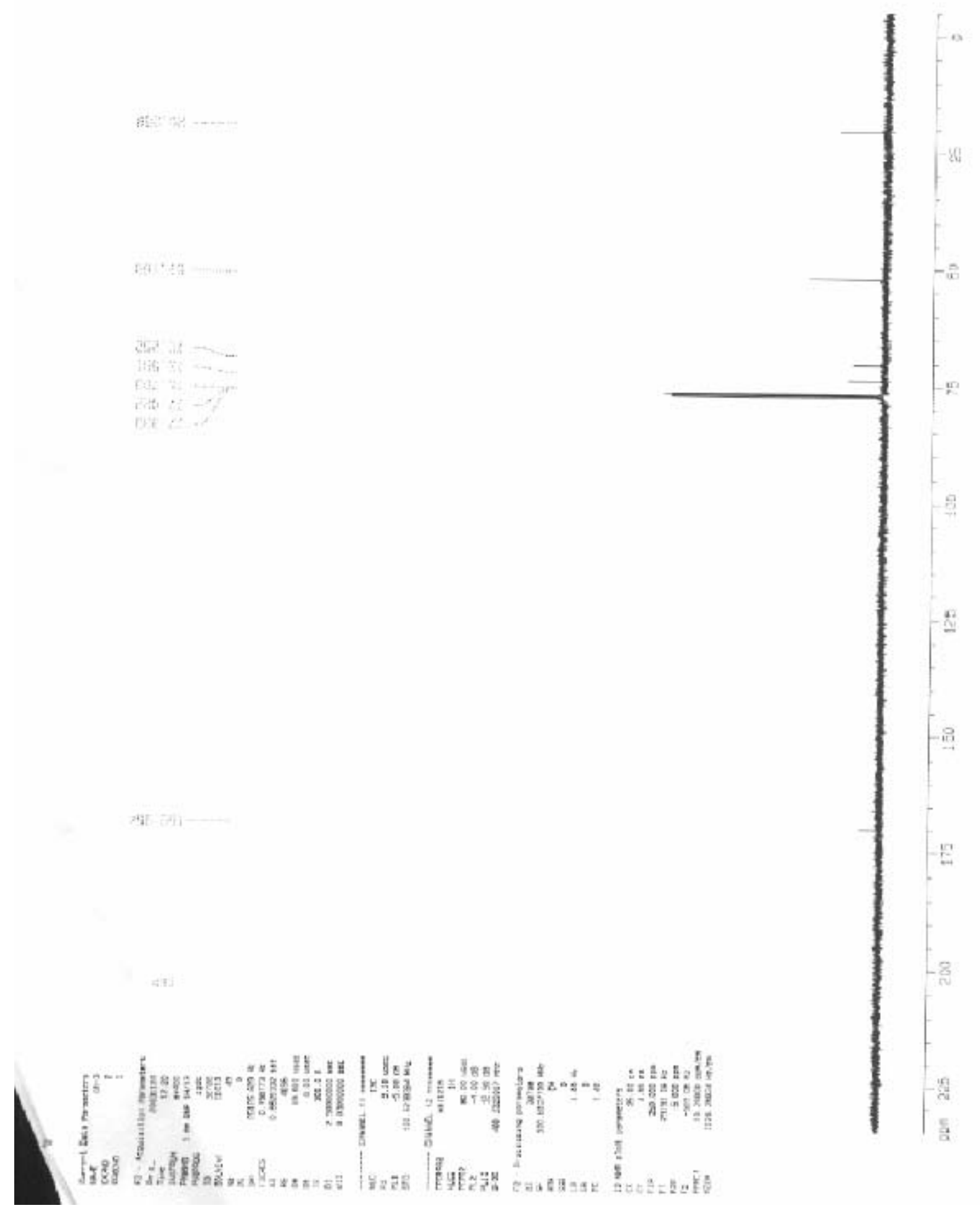


1-(2-(4-nitrophenyl)ethynyl)benzene (4)
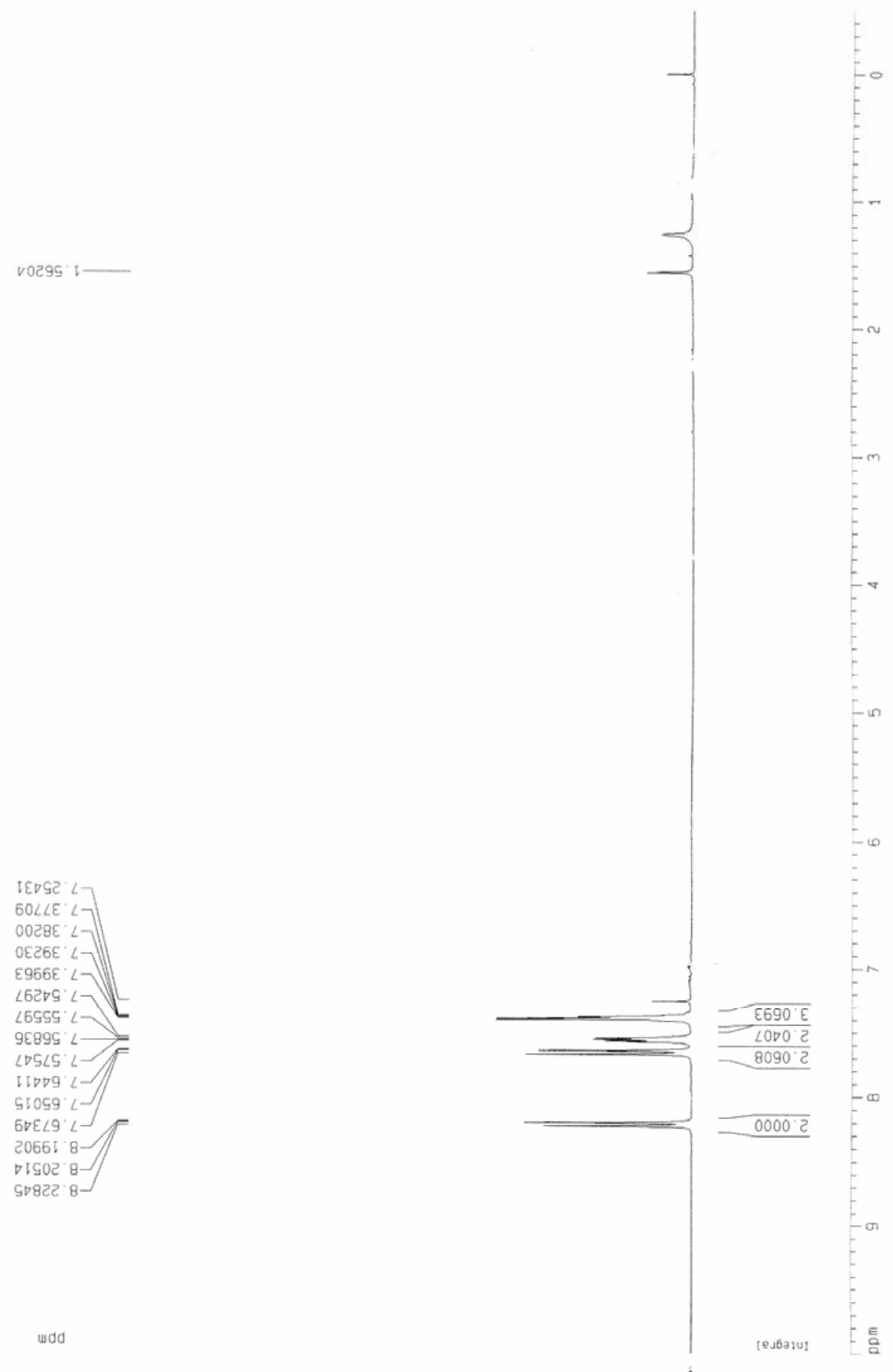
1-(2-(4-nitrophenyl)ethynyl)benzene (4)

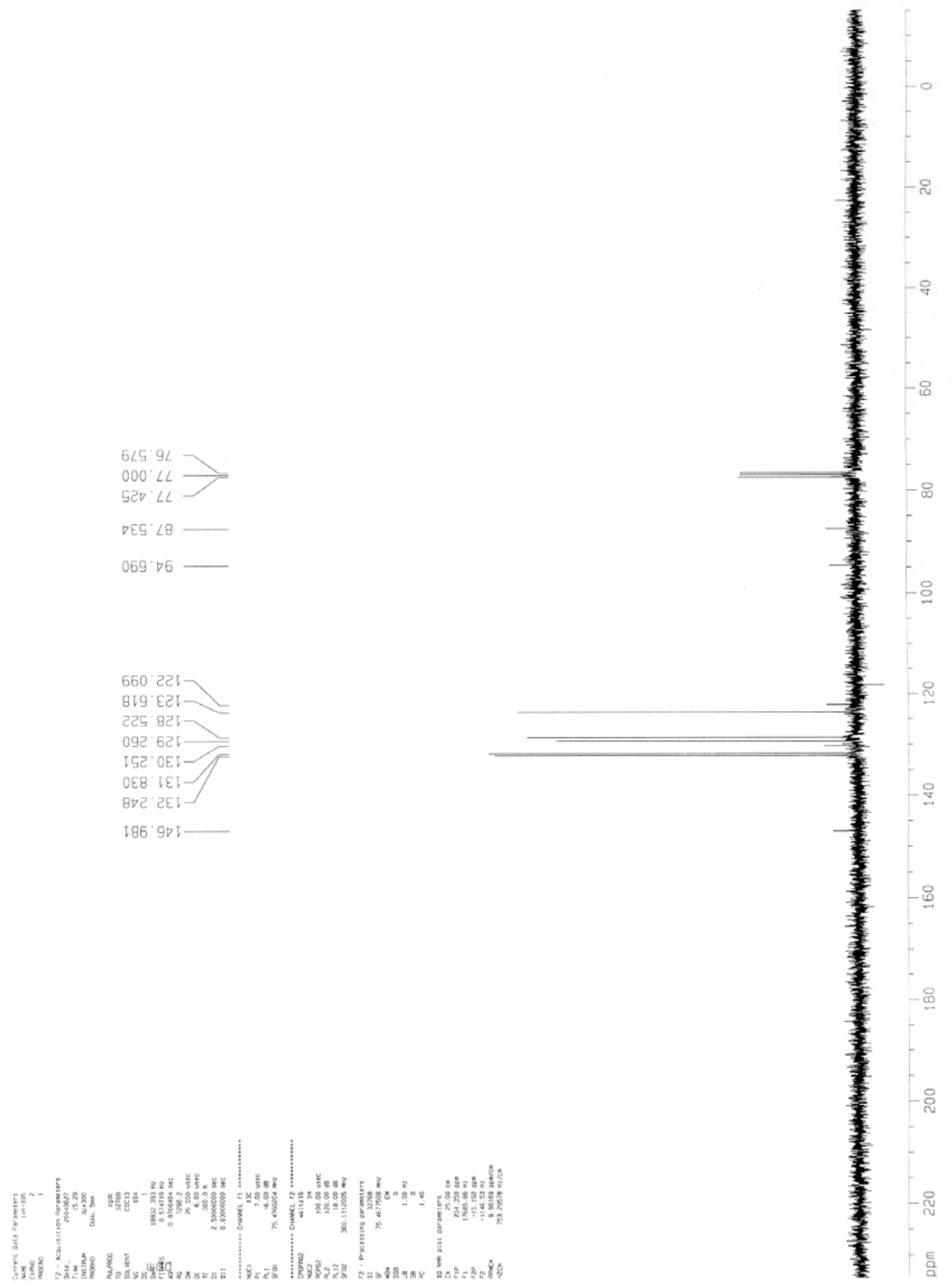


1-(dec-1-ynyl)-4-nitrobenzene (5)
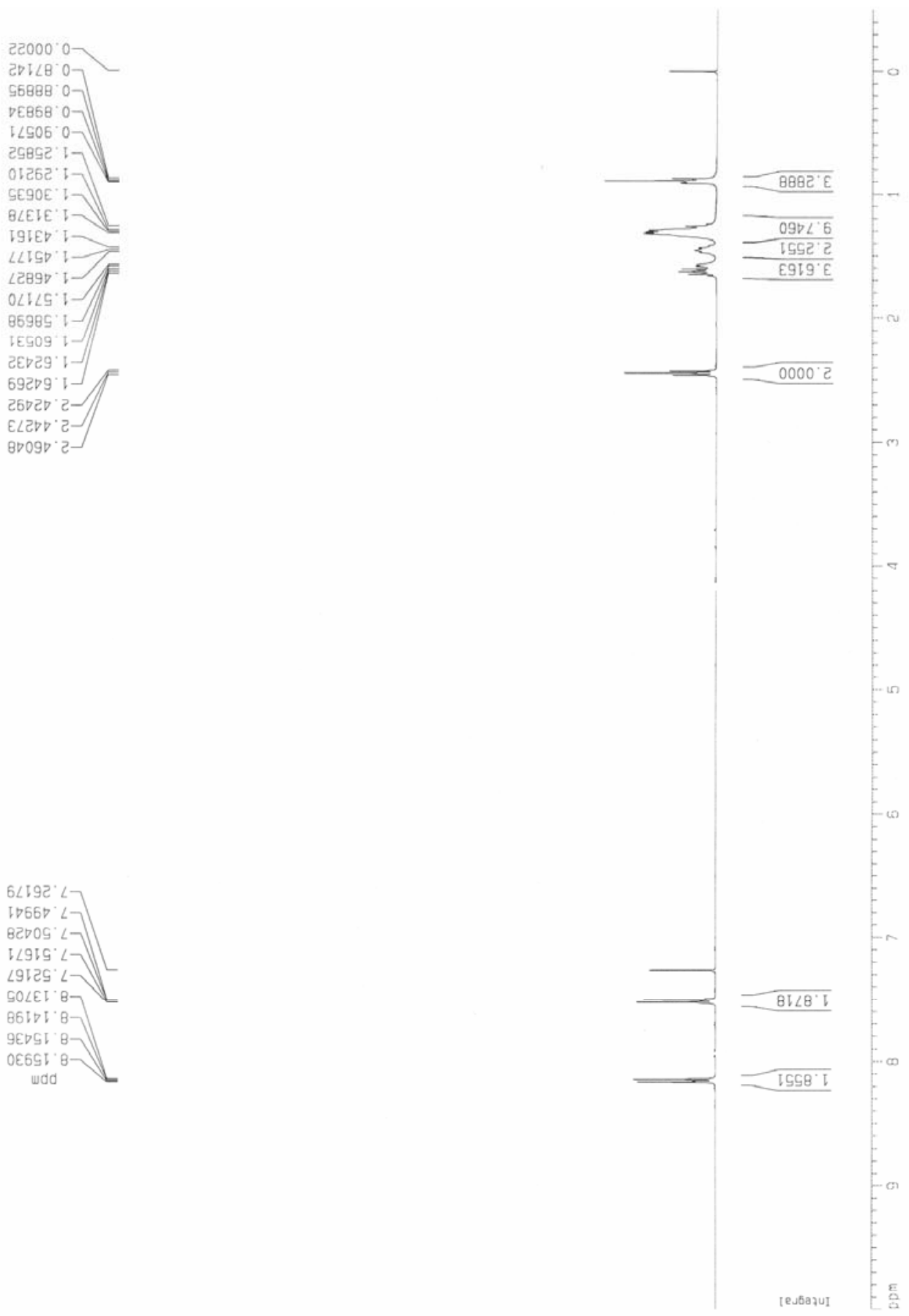
1-(dec-1-ynyl)-4-nitrobenzene (5)
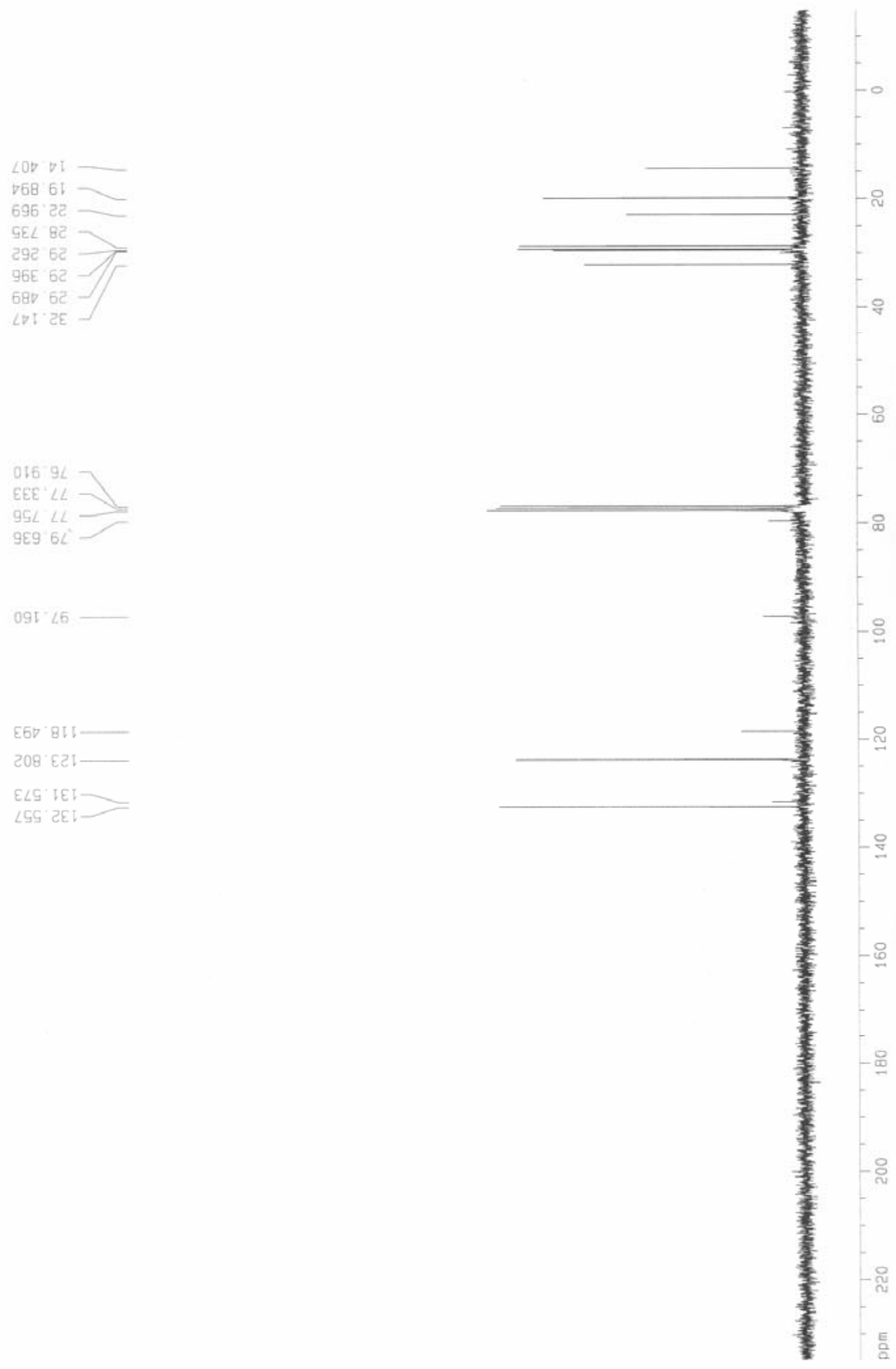
1-(2-(2-nitrophenyl)ethynyl)benzene (6)

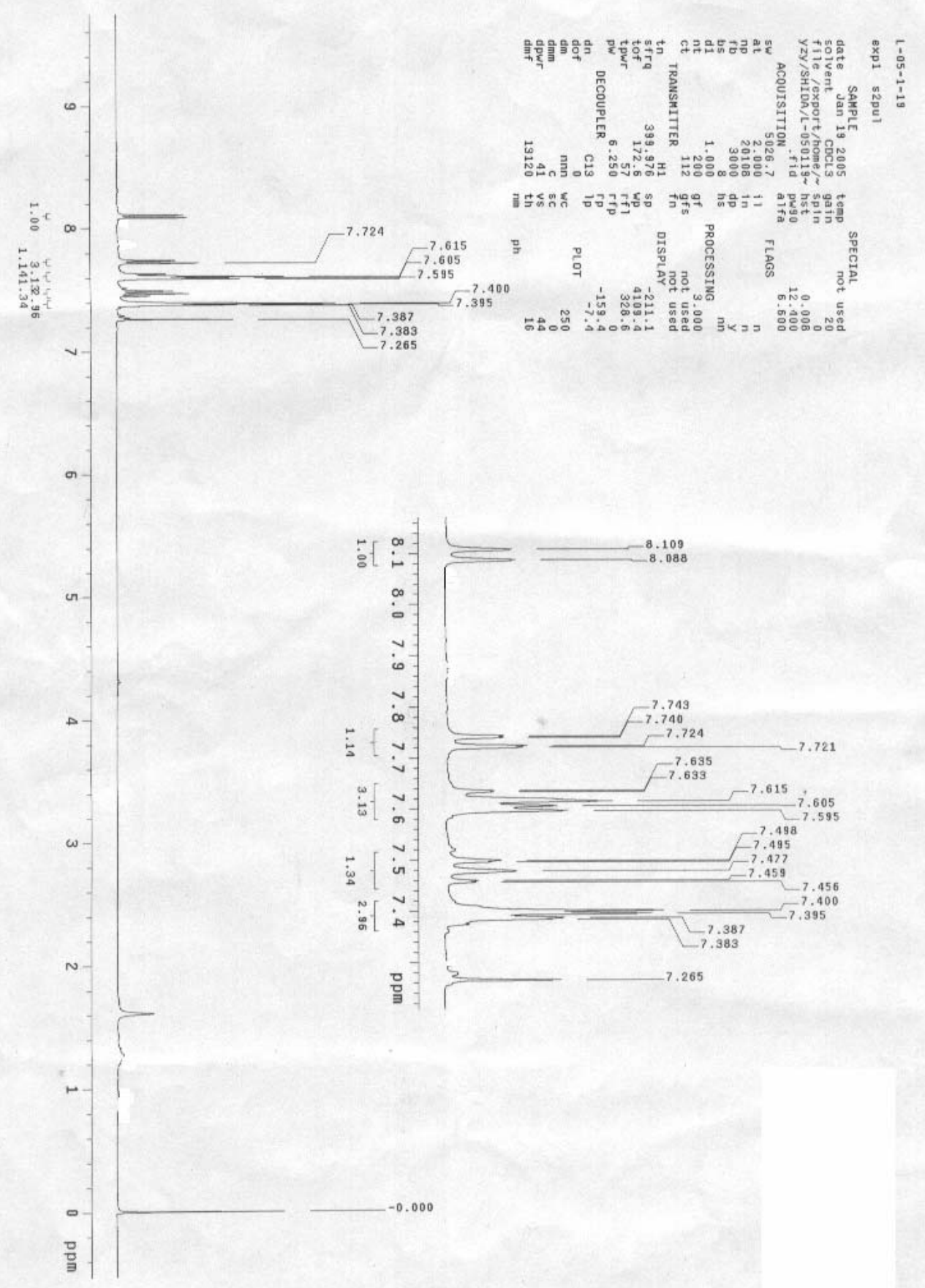


1-(2-(2-nitrophenyl)ethynyl)benzene (6)

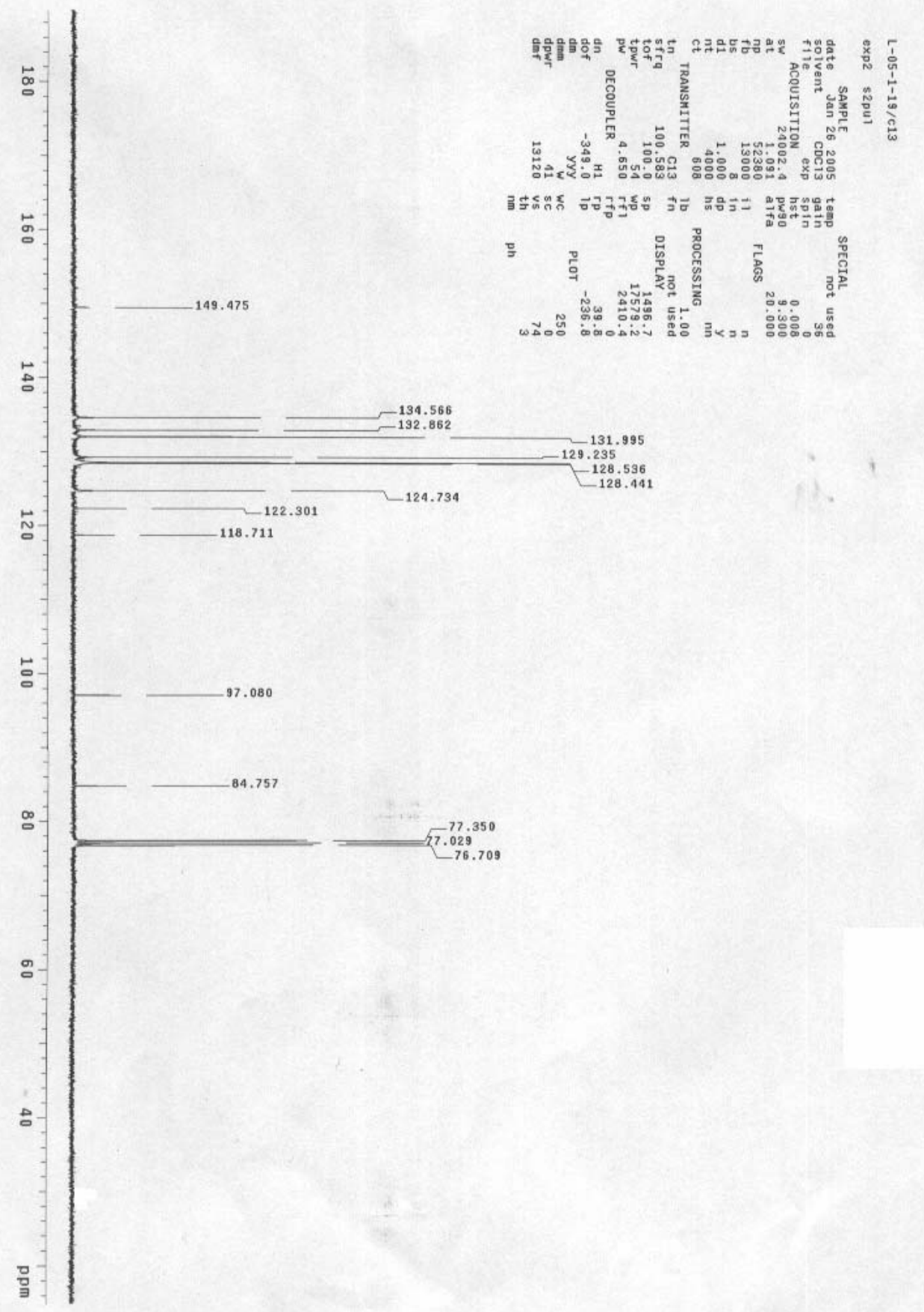


1,2-diphenylethyne (7)

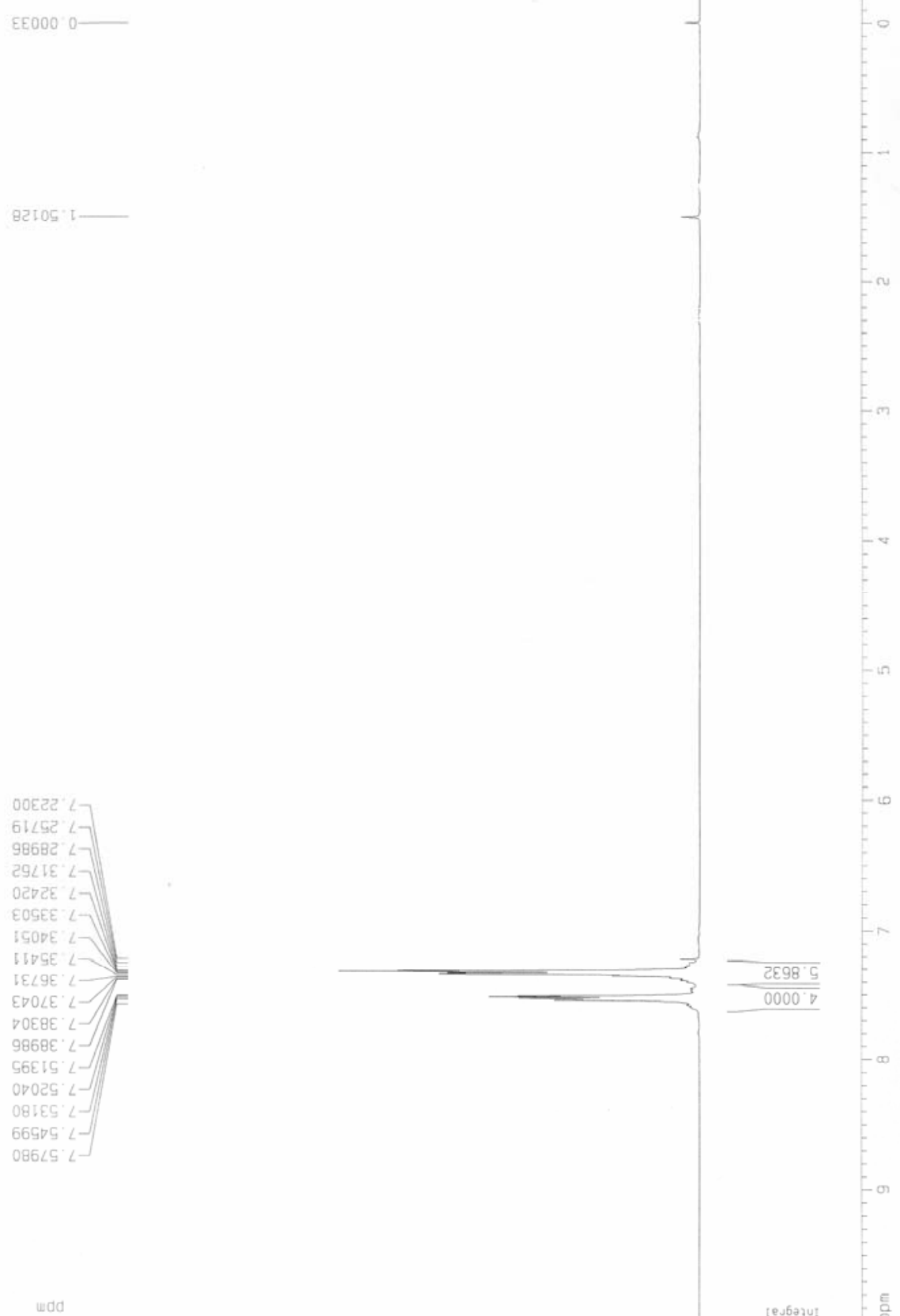


1,2-diphenylethyne (7)

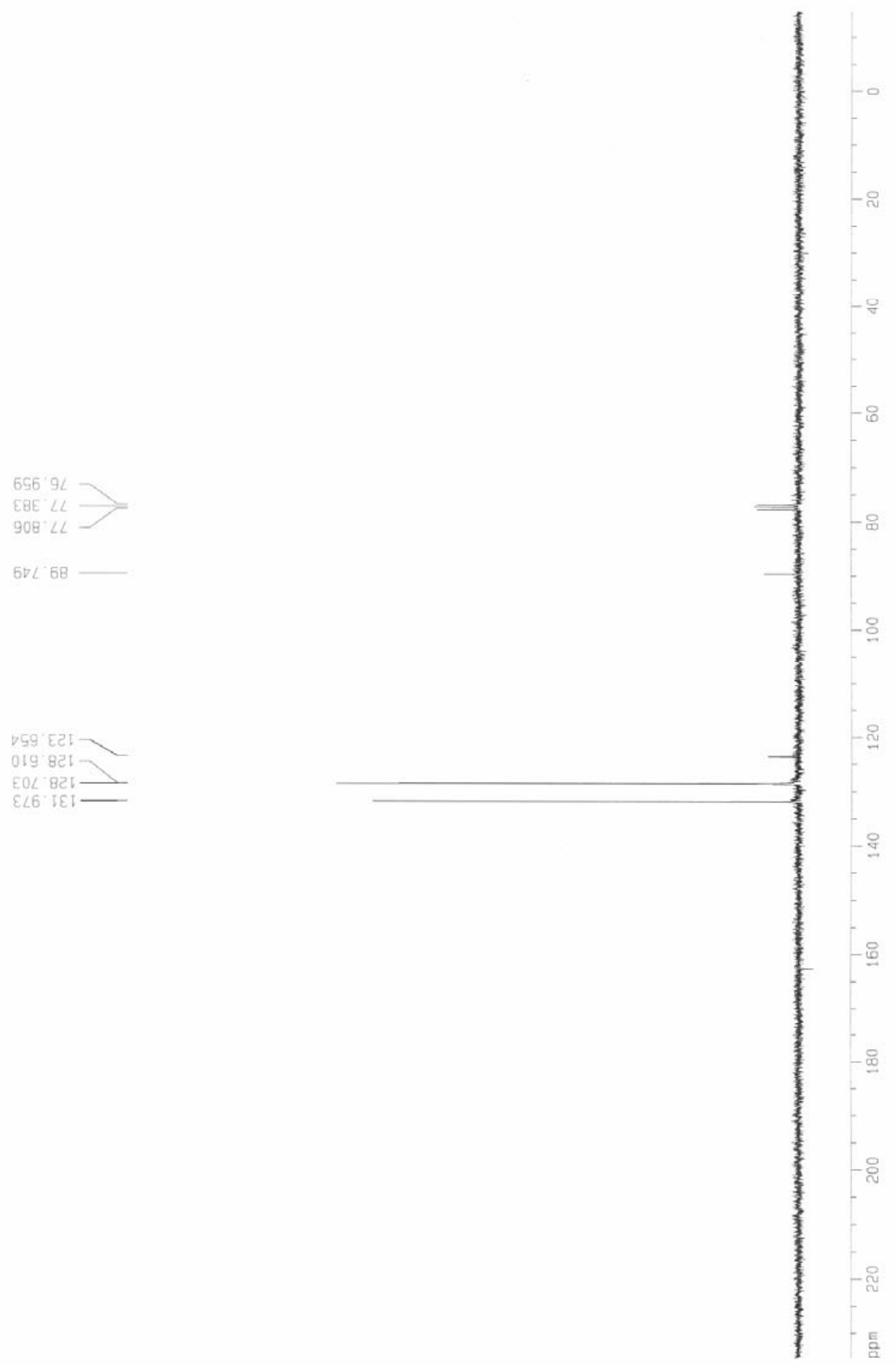


1-(2-p-tolylethynyl)benzene (8)

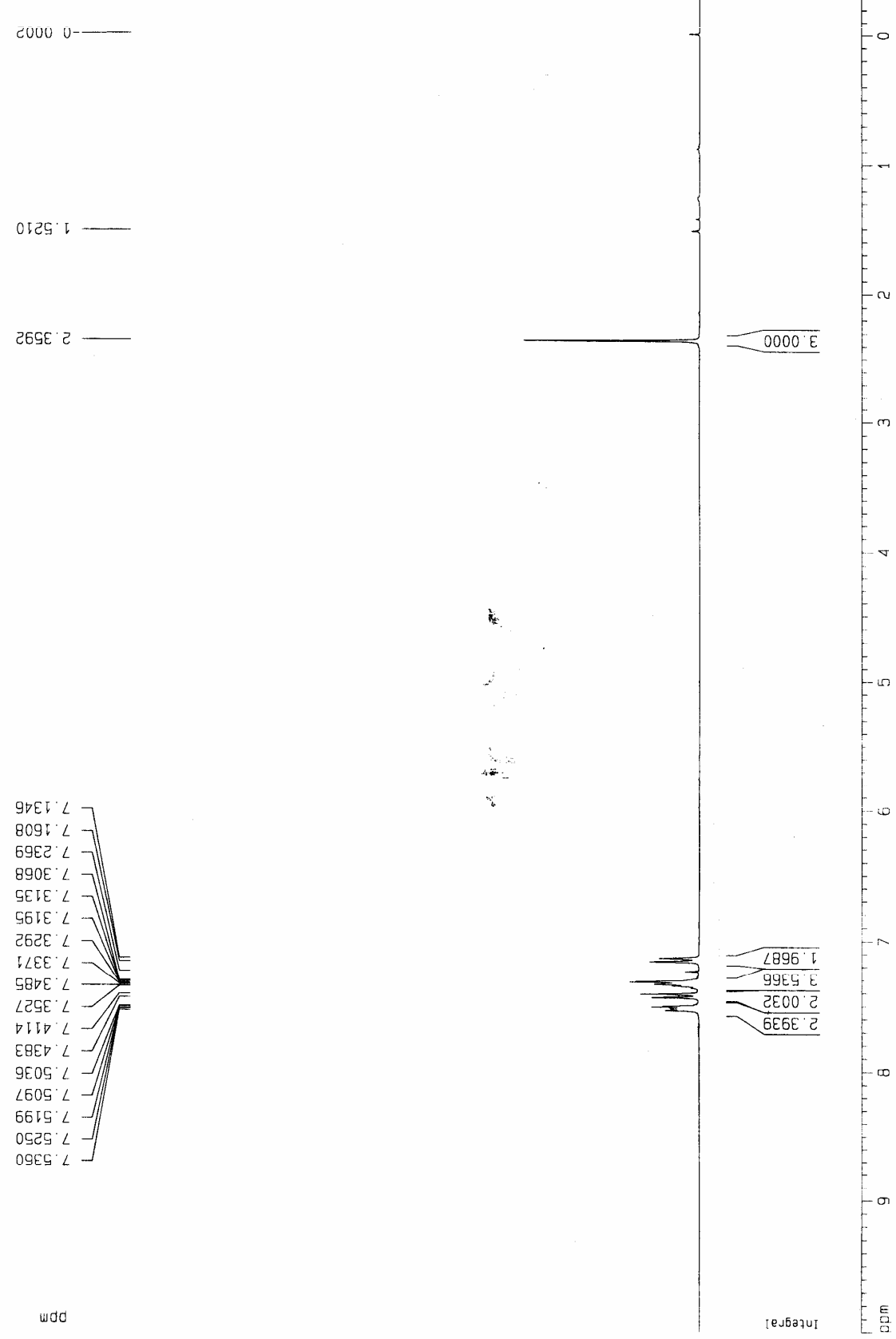


1-(2-p-tolylethynyl)benzene (8)

$198^{\circ}$

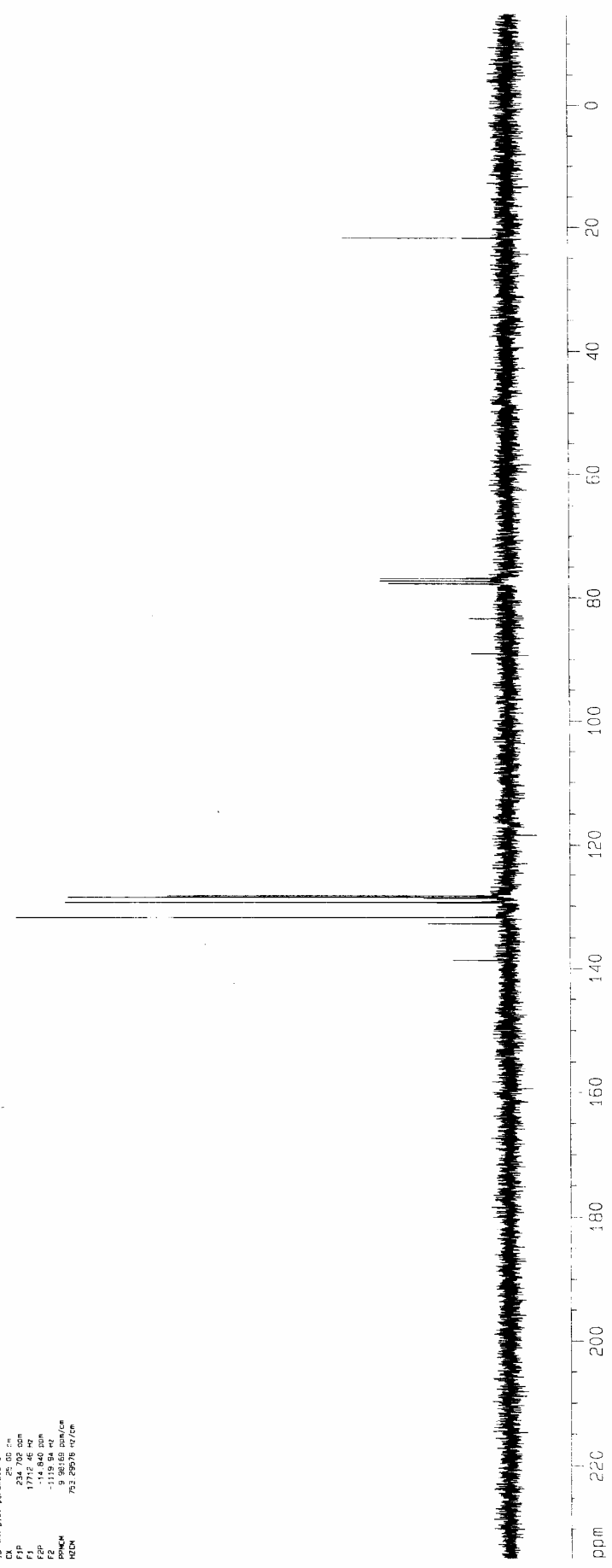


1-(2-o-tolylethynyl)benzene (9)
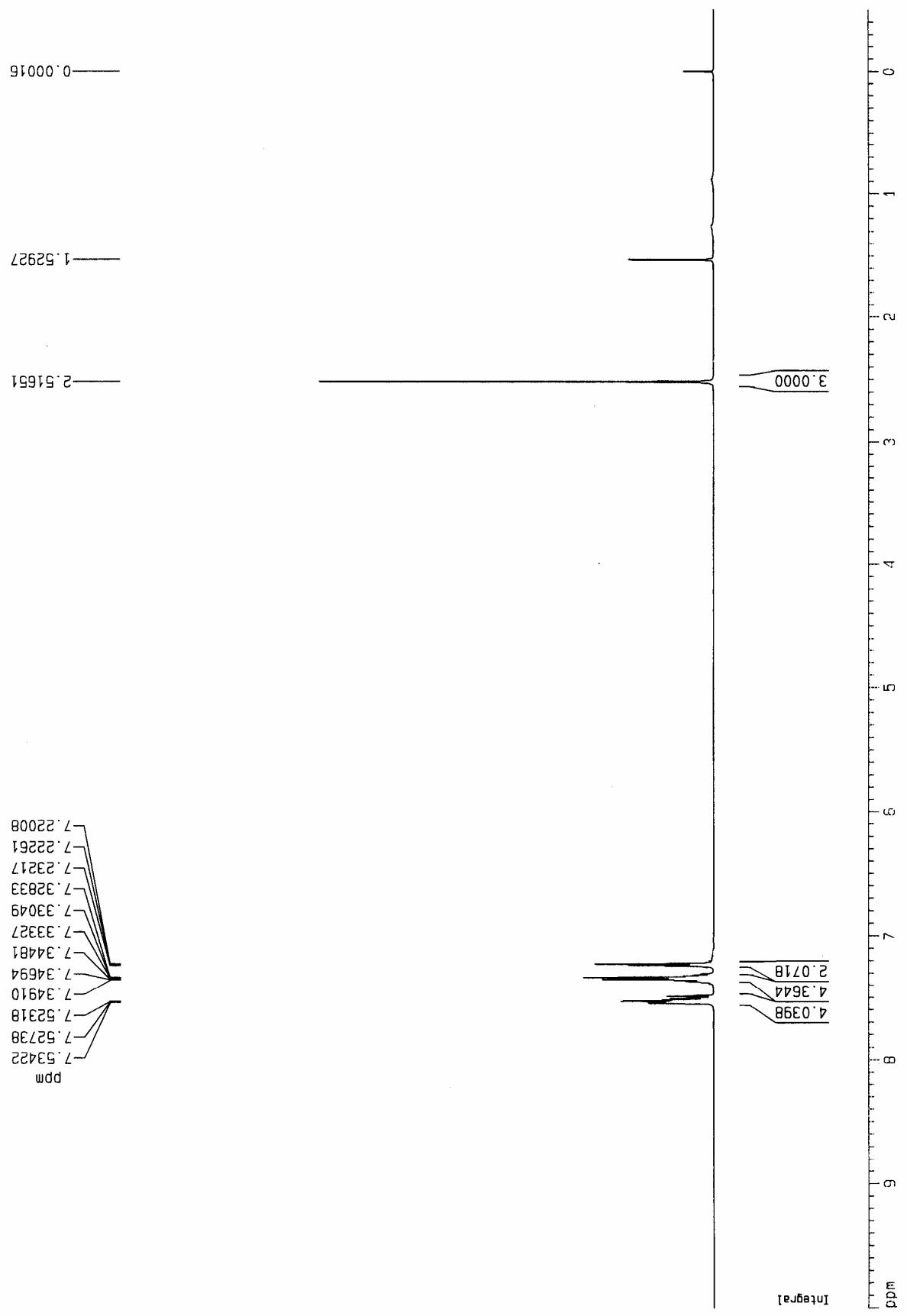
1-(2-o-tolylethynyl)benzene (9)
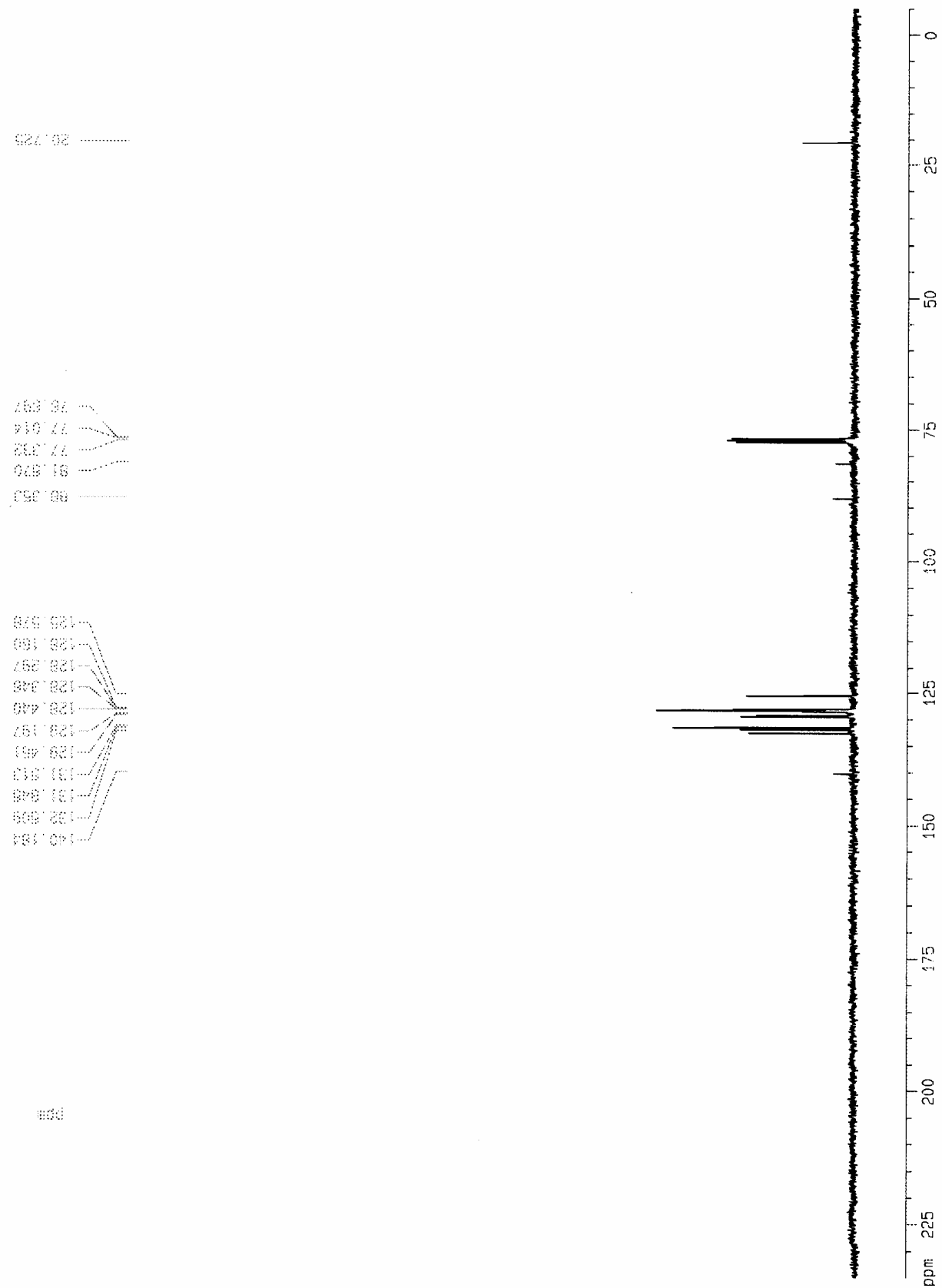


\section{1-methoxy-4-(2-phenylethynyl)benzene (10)}

$20000 \cdot 0-$

2EStS

$E D B 28^{\circ} \varepsilon-$

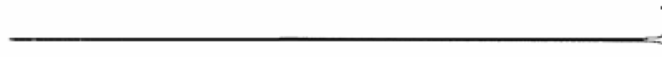

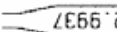

$90 \angle 98^{\circ} 9$

$16888^{\circ} 9$

$0655 e^{\prime} L$

GI8IE $\angle-$

GGट2E: $L$

ODSEE $L$

D2090. 4 -

$\varepsilon \nabla 58 \sigma^{\circ}\llcorner-$

$\triangle 6860^{\circ}:-$

$6 \angle 209^{\prime}<-$

$96909^{\circ}<-$

L8919' $<$

$16 \angle 15 . \angle-$

98ट25: $\angle$

wdd
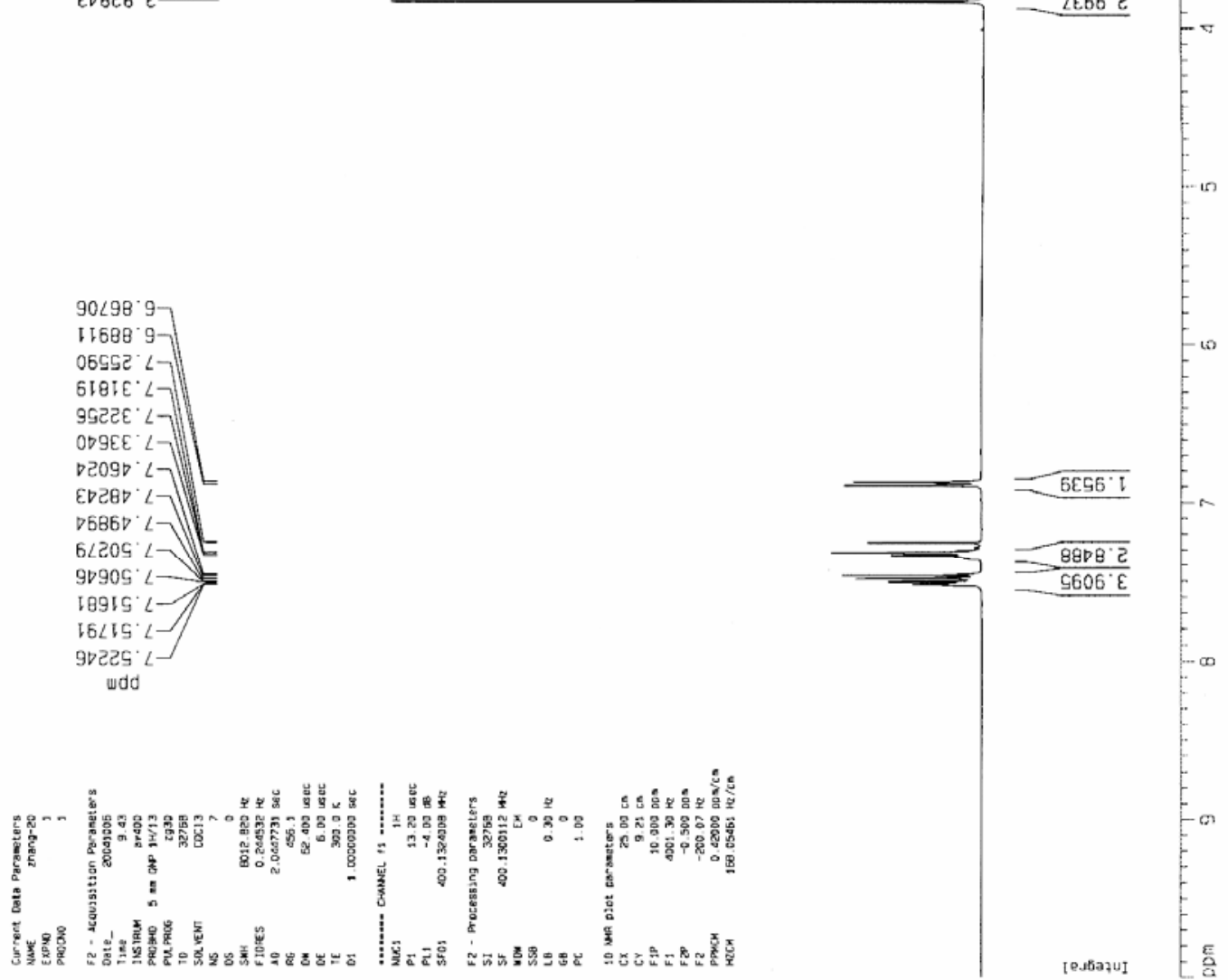
1-methoxy-4-(2-phenylethynyl)benzene (10)

$\angle B C \cdot 9{ }^{\prime}$

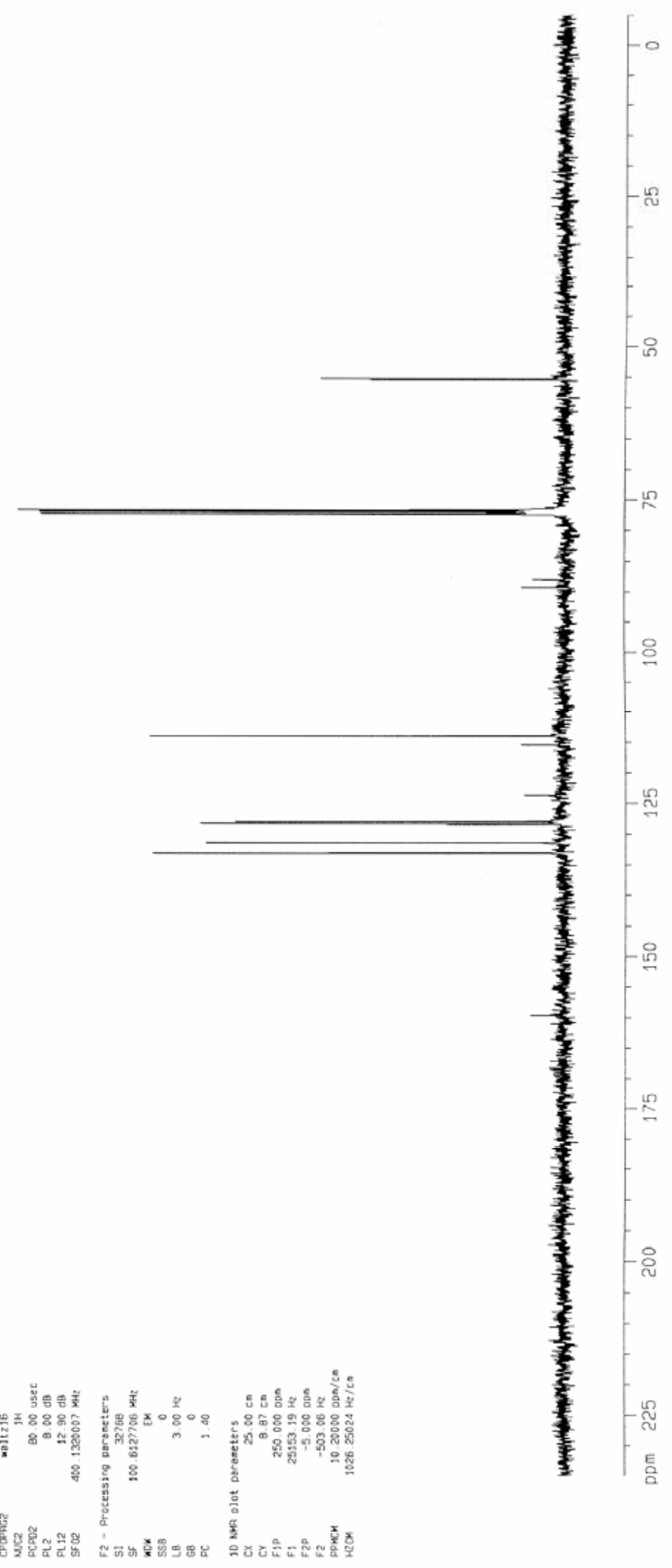

